\title{
Functional/Thermal Verification and Validation of an S-Band Radio for the Nanosatellites
}

\author{
Yaseen Zaidi ${ }^{1}$ \\ French South African Institute of Technology (F'SATI) \\ Dept. of Electrical, Electronic and Computer Engineering \\ Cape Peninsula University of Technology \\ Bellville 7530, South Africa \\ Norman G. Fitz-Coy and Robert van Zyl \\ Dept. of Mechanical and Aerospace Engineering \\ University of Florida \\ Gainesville, FL 32611, USA \\ and \\ French South African Institute of Technology (F'SATI) \\ Department of Electrical, Electronic and Computer Engineering \\ Cape Peninsula University of Technology \\ Bellville 7530, South Africa
}

\begin{abstract}
An S-band radio designed with commercial-grade components for the nanosatellites is functionally and thermally characterized for quiet transmission. The QPSK modulation impairments are minor over $-20^{\circ} \mathrm{C}$ to $+50^{\circ} \mathrm{C}$ at $24,26,28$ and $30 \mathrm{dBm}$ RF levels. The channel response is linear in error vector magnitude, frequency, phase, amplitude and IQ errors. On the average, the stability of amplifier bias and nonlinearity gives $-22 \mathrm{dBc}$ maximum upper/lower adjacent channel power and $1.27 \mathrm{MHz}$ occupied channel bandwidth. The acceptable level test results provide good confidence toward robust space-toearth transmission in variable solar weather at low earth orbital altitudes.
\end{abstract}

Keywords: Adjacent Channel Power (ACP); Error Vector Magnitude (EVM); Linearity; Nanosatellite; Nonlinear; Occupied Channel Bandwidth

Email addresses: yaseen.zaidi@ieee.org (Yaseen Zaidi), nfc@ufl.edu, vanzylr@cput.ac.za (Norman G. Fitz-Coy and Robert van Zyl )

${ }^{1}$ Corresponding author 
(OCBW); Power Amplifier (PA); QPSK; Radio; S-band; Thermal.

\section{Introduction}

The standard communications platform in the cube and nanosatellite missions is UHF/VHF system (Bouwmeester \& Guo, 2010; Selva \& Krejci, 2012) with deployable mono or dipole antenna. However, there is significant push from the users community for higher space-to-earth bit rates to receive more scientific data or higher resolution imagery. The International Telecommunication Union's amateur satellite band, the S-band, operating at $2400 \mathrm{MHz}-$ $2450 \mathrm{MHz}$ has attracted the attention of nanosatellite developers for increased performance in higher bandwidth and transmit power levels. One clear advantage of using S-band over UHF/VHF is the circularly polarized radiation with a smoother antenna pattern which induces less fading in the channel than in otherwise transmit and receive antennas at a mismatch in polarization (Nascetti et al., 2015). Secondly, the structure of S-band antenna can be patched (Fig. 1) on the satellite face pointing the nadir, thereby reducing the risk of in-orbit mechanized deployment of the antenna, failure of which could jeopardize the mission, partially or fully. This benefit imposes a serious constraint on the platform stability i.e., to ensure antenna pointing accuracy, typically done using momentum and reaction wheels or other attitude control hardware. So robust communication comes at the price of system reliability. The transition to the S-band based on-board communication systems design usually requires higher $\mathrm{DC}$ and $\mathrm{RF}$ power than the predominant $\mathrm{UHF} / \mathrm{VHF}$ systems.

In the present paper, functional verification and thermal validation of an S-band microwave transmitter (STX) designed for the nanosatellite ${ }^{2}$ applications is reported for performance. Shown in Fig. 1, the transmitter built with the Commercial Off The Shelf (COTS) parts, can be used as a communication subsystem for spacecraft health monitoring or as part of the payload for high speed download of the mission data to the ground station. It is observed that the variation in several transmission impairments in the transmitter are only marginal at $24 \mathrm{dBm}, 26 \mathrm{dBm}, 28 \mathrm{dBm}$ and $30 \mathrm{dBm}$ transmitting powers over $70^{\circ} \mathrm{C}$ span. This is confirmed by testing several facets of the transmission quality. The steadiness of digital modulation during transmission and

\footnotetext{
${ }^{2}$ In this paper the term nanosatellite refers to nano- and CubeSat classes of satellites.
} 

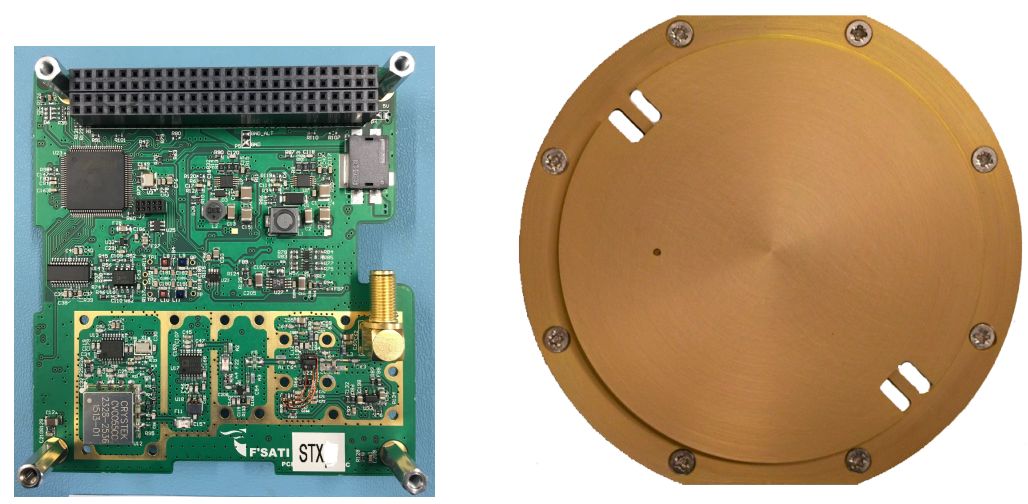

Figure 1: S-Band Transmitter (STX) and patch antenna in $10 \times 10 \mathrm{~cm}$ CubeSat standard form factor.

the stability of magnitude, frequency and gain imbalance have been verified in a single thermal cycle over $-20^{\circ} \mathrm{C}$ to $+50^{\circ} \mathrm{C}$, in steps of $10^{\circ} \mathrm{C}$ increment or decrement, and at 10 min dwell time. The conversion of DC to RF power is in the linear operation region of the Power Amplifier (PA). The cumulative time of - and + temperatures, respectively, agree well with the eclipse fraction usually $30-36 \%$ of the orbit period and the larger sunlit period at the popular orbital altitudes $(550 \mathrm{~km}$ to $600 \mathrm{~km})$ in the Earth Observation (EO) missions. The test dwell time, 10 min, exceeds the contact period of the satellite with the ground station. From horizon to horizon, typical EO passes last $11 \mathrm{~min}$ to $14 \mathrm{~min}$ and the usable communication window, which includes acquiring and establishing the link, is a fraction of the duration of satellite visibility to the ground station. Therefore, the higher transmitted $\mathrm{dBm}$ levels can be used to set margins in the link budget at low elevation angles when the slant range is the highest and thus the free space path loss, too. Additionally, higher power margin in the transmitter are useful in spaceto-earth fade due to the atmospheric effects and especially rain attenuation. The thermal cycle time roughly correlates to the period of Sun-Synchronous Orbit (SSO), which is the preferred orbit in the EO missions due to ideal illumination conditions for imaging at the equatorial crossings.

\section{Heat-Limited Acceptance Level Thermal Testing}

Using commercial grade components in nanosatellite missions is a venturous undertaking for their exposure to harshness of the space weather and 
especially of regular heating and cooling during orbit propagation. With the COTS technology operating in the space environment for which it is not rated, the acceptance level testing, although small in scope, serves as the basis of qualified confidence that the flight hardware shall withstand the ambiance of space and perform as required.

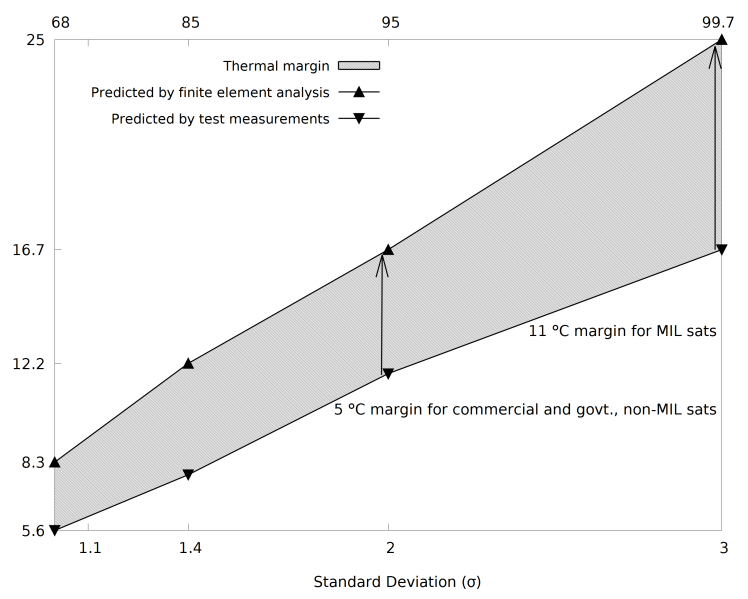

Figure 2: Thermal uncertainty margins based on 68-85-99 rule and adopted in MIL-STD-1540 (Gilmore., 2002).

Among the environmental loads on the flight hardware, the mechanical vibration is short lived i.e., only acting on the satellite in-situ the rocket launch. Once in-orbit, the damage due to the radiation bombardment is probabilistic. The thermal loads are periodically invariable. In an SSO, a nanosatellite coverage typically has 14 to 17 orbits a day. In each lap, if no abrupt variation in solar weather occurs, then the dynamic thermal loading on the spacecraft can be considered deterministic and enforceable for a fixed duration. The recurring of thermal loads at regular intervals continues during the mission life, e.g., in a 3 year mission as many as 16000 thermal cycles will be endured. In general, the high-end missions use $11^{\circ} \mathrm{C}$ thermal design margins (Gilmore., 2002). In designs with a $5{ }^{\circ} \mathrm{C}$ thermal margin which is a common practice in commercial missions, it is $2 \sigma$ or $95 \%$ probable that the hot and cold temperature extremes in the spacecraft will fall within the limit as shown in Fig. 2. The margins are applied after validation of the Finite Element (FE) thermal model of the spacecraft with the results of the thermal balance test. Obviously, high margins is stringent requirement considering the inherently risky nanosatellites. Typical nanosatellite projects 
do not enjoy the luxury of long schedule and high funding, after all low cost design, liberal systems engineering and thus running under risks is the underlying philosophy of any nanosatellite program. Generally, a detailed thermal simulation with elaborated CAD geometry, finer mesh, junction temperatures of integrated circuits, as well as the thermal and radiative properties of the spacecraft assemblies, is compromised. It follows that in a passive thermal control scheme, the thermal cycle testing profoundly substantiates itself toward the success of a nanosatellite mission, even though the other environmental tests are chanced or disregarded (Mengu et al., 2012).

In this paper, the thermo-functional verification and validation has the following fair specification:

1. Loads are heat-limited; applied at acceptance level criteria, in steps of $10^{\circ} \mathrm{C}$ increment or decrement, from $-20^{\circ} \mathrm{C}$ to $+50{ }^{\circ} \mathrm{C}$ at dwell time $10 \mathrm{~min}$ in each step.

2. Vacuum is absent, the combined heat transfer mechanism is conductive and convective. In a real Concept of Operation (ConOp), the transfer will be conductive and radiative.

3. Each test is performed at a fixed RF power level similar to an actual ConOp scenario where the transmission will likely be at constant power in a single pass of the satellite.

\section{Signal Design in the S-band Transmitter (STX)}

The STX is a compact S-band transmitter compatible with the CubeSat Kit PC/104 form factor (Fig. 1). The STX operates in the amateur Sband $2400 \mathrm{MHz}-2450 \mathrm{MHz}$ and STXC operates at $2200 \mathrm{MHz}-2300 \mathrm{MHz}$ in the commercial S-band. The output power is adjustable in $2 \mathrm{~dB}$ steps from $24 \mathrm{dBm}$ to $30 \mathrm{dBm}$. Data transmission rates of up to $2 \mathrm{Mbps}$ are supported (1 Mbps of user data and $1 \mathrm{Mbps}$ encoding) with $1 / 2,1 / 4$ and $1 / 8$ rate modes. The STX uses an open network encoding scheme based on the IntelSAT IESS308 specification which allows it to be used with low cost commercial satellite receivers. The STX uses either Quadrature Phase Shift Keying (QPSK) or Offset QPSK modulations. The architecture of QPSK modulator is shown in Fig. 3.

\subsection{Significance of Performance Parameters}

The main concern of this paper is two-fold. Firstly, the analysis of transmission impairments caused by left and the middle dotted blocks in Fig. 3, 
discussed in Section 4. The performance metrics (EVM, SNR, $\rho$ and IQ based parameters) related to modulation blocks result in in-band distortion. This type of distortion impairs the correlated components in the signal content which results in confusing the ground station receiver in correctly deciding the actual values in the binary stream. Incorrect decisions ultimately result in low Signal to Noise Ratio (SNR) and poor Bit Error Rate (BER), and low $E_{b} / N_{0}$, the important link budget parameter.

Secondly, we analyze the impairments caused by the dotted block to the right, discussed in Section 5, which pertains to nonlinearity and efficiency. This block results in out-of-band distortion in the signal content which has uncorrelated components causing the interference in the wanted signal. The effect of amplifier nonlinearity on the QPSK system would be that there will be waste of DC power on-board the spacecraft regarded as its lifeline. There will be unwanted heat generation while the RF conversion in transmission will not increase. Moreover, some signal energy will lie outside the in-band which could result in on-board susceptibility or interference to the radio systems operating near the ground station.

The aspects of linear modulation accuracy and nonlinear distortions are validated for drift with extensive thermal testing and thus a more complete picture of the system distortions is obtained. In the current section, we examine the theoretical foundation of the measurements across the signal path.

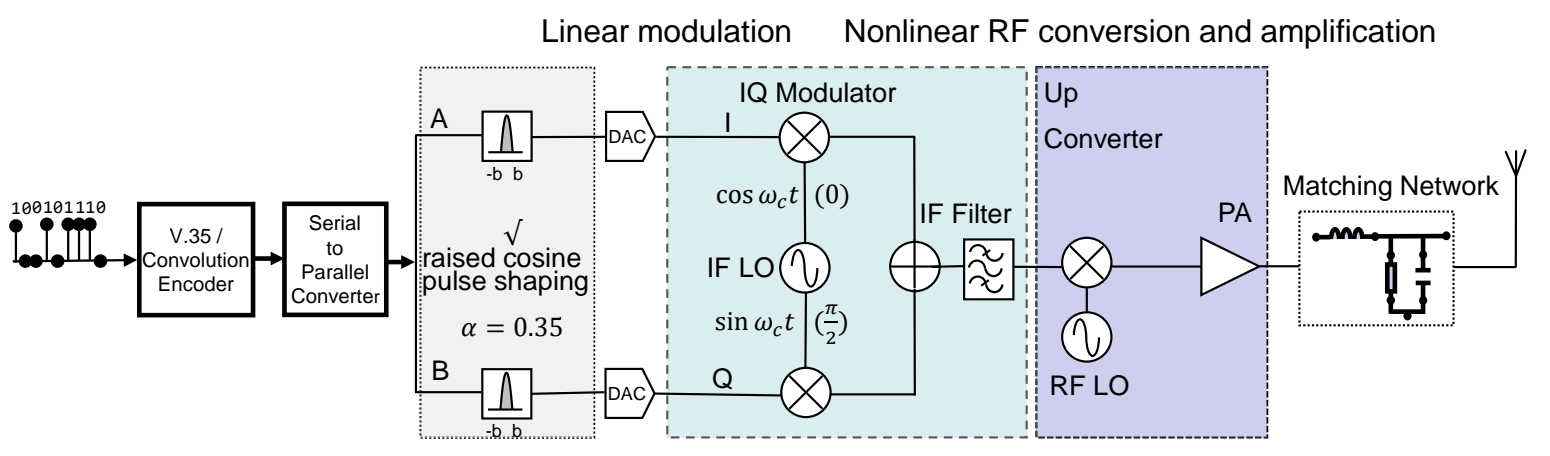

Figure 3: Linear (in-band) and nonlinear (out-of-band) impairment sources in the RF chain of the on-board transmitter. 


\subsection{Band Limited Throughput}

In any digital wireless application, bandwidth efficiency is usually a major worry due to spectrum congestion in channel spacing. There are two major aspects of bandwidth efficiency in a QPSK system.

Firstly, to maximize the transmission rate, the signal pulses are modeled to occupy the smallest bin of frequency by means of a pulse shaping filter. The binary pulses are band limited by approximating the ideal sinc function, given in Eq. 1, in an anti-Inter Symbol Interference (ISI) Nyquist filter, the root raised cosine ( $r r c$ ) filter, as illustrated in Fig. 3. Full raised cosine amplitude is obtained by applying another $\operatorname{rrc}$ filter at the receiver in the satellite ground station.

$$
\operatorname{rrc}(t)=\frac{\sin \pi t / T}{\pi t / T} \cdot \frac{\cos \pi \alpha t / T}{1-4 \alpha^{2} t^{2} / T^{2}}
$$

The right part in Eq. 1 damps the power in the pulse thus reducing the interference. When $\alpha \rightarrow 0$, the roll-off is steeper, ISI is lesser and the Adjacent Channel Power (ACP) and Occupied Channel Bandwidth (OCBW) also lower. In the real world, OCBW is always higher than the symbol rate due to non-zero $\alpha$.

Secondly, the bandwidth is halved by splitting even $m$ and odd $n$ numbered bits. Two consecutive bits give an output at the quadrature summation $x(t)=b_{m} A_{c} \cos \omega_{c} t-b_{n} A_{c} \sin \omega_{c} t$. For integers $m$ and $n$, if $m^{2} \neq n^{2}$, in any interval $0<t<\pi$, due to two phases lagging, the overlapping is

$\int_{0}^{\pi} b_{m} A_{c} \cos \omega_{c} t \cdot b_{n} A_{c} \sin \omega_{c} t d t=0$ and hence both streams can be distinctly distinguished at the satellite receiver on the ground. Four possible symbol states are 11, 10, 01 and 00 corresponding to, respectively, $\pi / 4,-\pi / 4,3 \pi / 4$ and $-3 \pi / 4$ phases i.e., the vertices of the constellation square.

Both operations ramify a number of impairments which mainly result in digression in the IQ amplitude or phase or both. The skew in the ideal and the actual constellations is measured as Error Vector Magnitude (EVM).

\subsection{Linear Detection}

If the two consecutive symbols on paths $\mathrm{A}$ and $\mathrm{B}$ happen to lie in the diagonally opposite quadrants in the IQ plane, the resulting symbol waveform exhibits discernible variation in the amplitude corresponding to the large phase transition. This effect manifests itself in the output signal as a variable envelope in which the amplitude varies in linear proportional to the variation in the phase. The amplitude appears to have discontinuity-like sudden jumps 
at the phase changes. In such a signal, the model $x(t)$ has a linear timevarying $A_{c}(t)$ envelope which also varies in linear proportion to phases $\cos \omega_{c} t$, $\sin \omega_{c} t$. After multiplexing, the linearities must still hold. Hence, the linear QPSK modulation is hugely sensitive to the nonlinear distortions.

The design and test goal is to ensure transmission with only inappreciable nonlinearities.

\section{Modulation Accuracy and Impairment Measurements}

A testbed is developed for vector measurements (Roblin, 2011). The setup consists of a STX board connected to Agilent EXA signal analyzer, microwave signal generator and power supplies. While in the thermal chamber by Binder, as shown in Fig. 4, an Arduino based dashboard configures the STX by setting up the baseband FPGA registers and transmit power levels. The STX RF output is connected to the signal analyzer input through a $30 \mathrm{~dB}$ high power attenuator. Vector Signal Analysis (VSA) software is used as a wideband sampling front end. The insertion loss of the cable and attenuator was accurately measured and accounted for in the power measurements. All tests are done on QPSK, at full data rate and standard $50 \Omega$ load and normally with pulse shaping enabled.

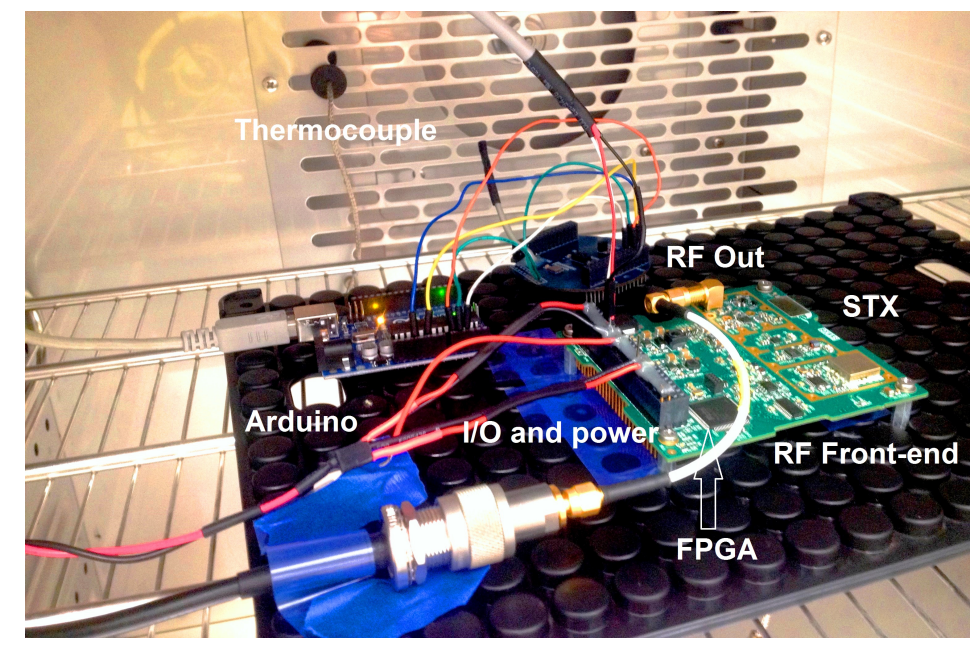

Figure 4: Placement of STX and the dashboard in the thermal chamber. 
TX output power (dBm)

at 30, 28, 26 and 24 power levels (top down)
TX currents (A) at $\mathrm{V}_{\text {bias }}=5 \mathrm{VDC}, \mathrm{V}_{\text {bat }}=7.2 \mathrm{VDC}$
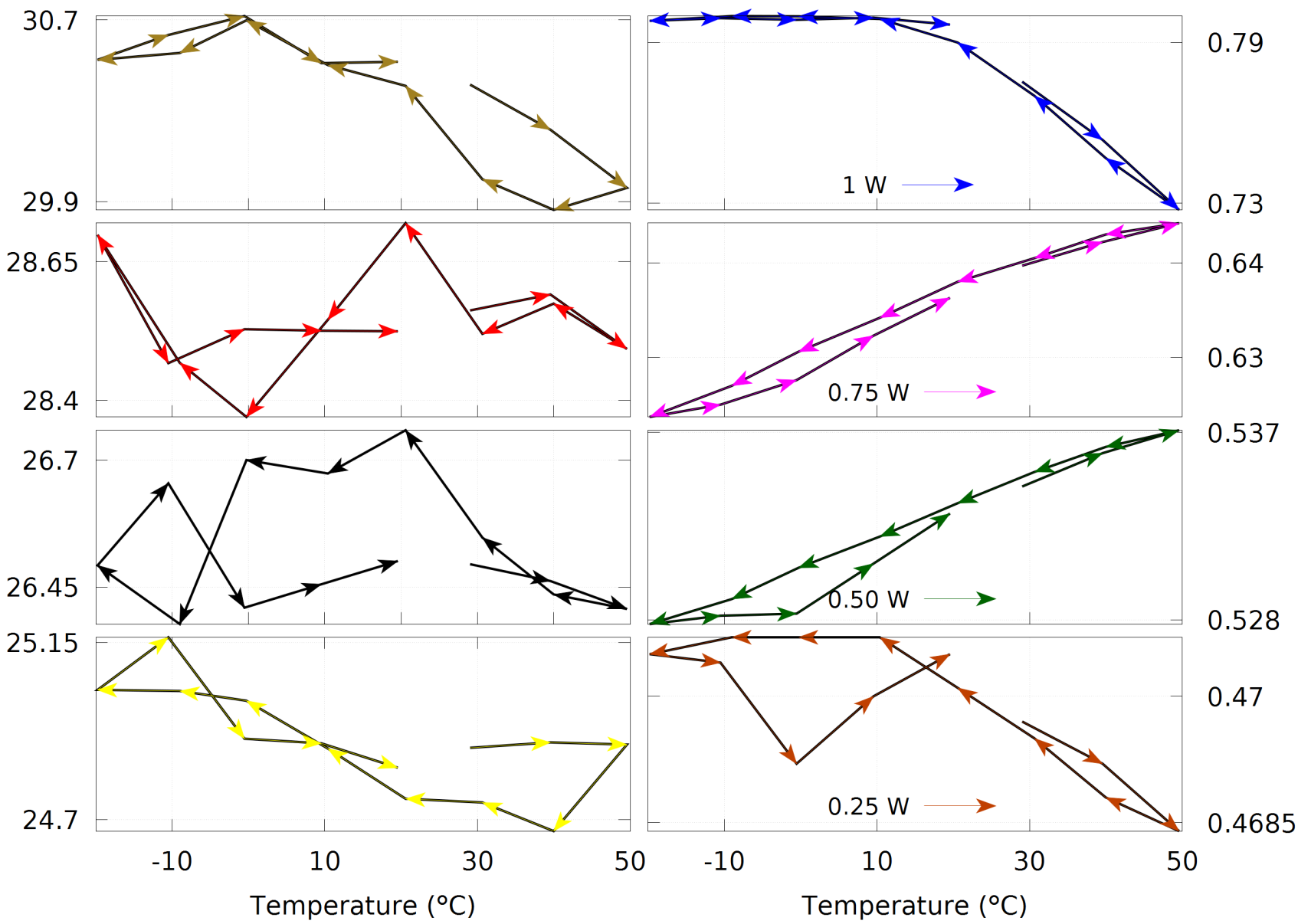

Figure 5: Characterization of STX radio operating at different RF power levels at $2.2 \mathrm{GHz}$ (left) and the corresponding currents (right) during thermal cycle.

\subsection{Channel Power and DC Power}

Fig. 5 illustrates the output RF power and DC current profile of the transmitter at four different levels over the temperature cycle. The variation in the output is $0.49 \mathrm{dBm}, 0.38 \mathrm{dBm}, 0.65 \mathrm{dBm}$ and $0.85 \mathrm{dBm}$ at respective $24 \mathrm{dBm}, 26 \mathrm{dBm}, 28 \mathrm{dBm}$ and $30 \mathrm{dBm}$ levels. The channel stability is supported by corresponding variation in transmitter currents: $2.3 \mathrm{~mA}, 9.3 \mathrm{~mA}$, $20.5 \mathrm{~mA}$ and $72.3 \mathrm{~mA}$. Notice the jump in DC variation (300\%) from $24 \mathrm{dBm}$ to $26 \mathrm{dBm}$ RF power and $250 \%$ from $28 \mathrm{dBm}$ to $30 \mathrm{dBm}$, while the variation 
at $26 \mathrm{dBm}$ to $28 \mathrm{dBm}$ is quiescent at $120 \%$. The RF performance may be expected to be stiller if power is switched in-orbit from $26 \mathrm{dBm}$ to $28 \mathrm{dBm}$.

The standard deviation in transmitter current is $0.7 \mathrm{~mA}, 3.2 \mathrm{~mA}, 6.9 \mathrm{~mA}$ and $24 \mathrm{~mA}$ and in RF power $0.1291 \mathrm{dBm}, 0.1217 \mathrm{dBm}, 0.0895 \mathrm{dBm}$ and $0.2766 \mathrm{dBm}$ at respective $24 \mathrm{dBm}, 26 \mathrm{dBm}, 28 \mathrm{dBm}$ and $30 \mathrm{dBm}$. From the stability of bias standpoint, the best operation mode is $24 \mathrm{dBm}$ at which the deviation is the least, whereas, from the conversion point of view the desirable range is $28 \mathrm{dBm}-30 \mathrm{dBm}$.

At low temperatures the output power levels still hold, therefore, the transmitter may be operated via battery in eclipse or during the night passes. At high temperatures, there tends to be a decline in output power, which is more prominent at higher power levels. This is a mix effect due to PA operation at the edge of linearity limit and wavering of the bias due to selfheating of the amplifier as well as the high ambient temperature.

\subsection{Error Vector Magnitude (EVM)}

After the preliminary exercise of proving STX performance in the transmitted output RF power, it is now convenient to seek insight in the transmission impairments and characterize them over the thermal cycle. The fundamental and composite figure of merit, encompassing several types of distortions in the I and Q, is the EVM (Noël \& Brousse, 2011).

For the measured signal $x_{2}(t)$, its distance, in discretized form, in the IQ plane over $n$ samples computed as rms or \% signal amplitude strength from the reference signal $x_{1}(t)$, is expressed as

$$
E V M_{r m s}=\sqrt{\frac{\sum_{n}\left|x_{2}(n)-x_{1}(n)\right|^{2}}{\sum_{n}\left|x_{1}(n)\right|^{2}}}=\sqrt{\frac{\sum_{n}\left(\Delta I_{i}{ }^{2}+\Delta Q_{i}{ }^{2}\right)}{\sum_{n}\left(I_{i}{ }^{2}+Q_{i}{ }^{2}\right)}}
$$

where $\sum_{n}\left|x_{1}(n)\right|^{2}$ is the normalized to the peak amplitude of the constellation (McKinley et al., 2004).

The top plot in Fig. 6 illustrates the rms EVM steadily rising with temperature. The variation is no more than $0.5 \%$, confirming low noise and high linearity. The bottom plot shows the peak EVM at different symbol rates. The variation is within 3\% over the temperature cycle. This is first indication of excellent modulation accuracy that shows that the in-band distortion is minimally affected by the thermal variation. 


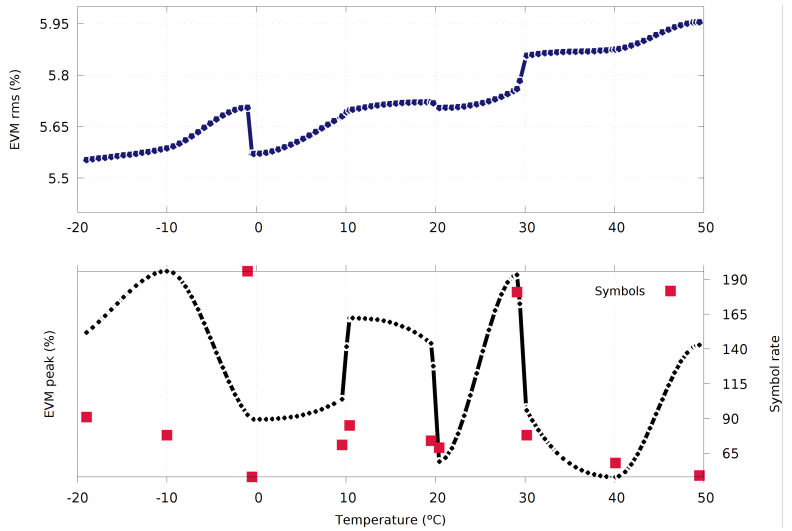

Figure 6: Variation in the rms and peak error vectors at $24 \mathrm{dBm}$ output.

\subsection{Signal to Noise Ratio (SNR) and Waveform Quality Factor}

It is often sought after to estimate other performance metrics from the EVM (Gharaibeh et al., 2004; Mahmoud \& Arslan, 2009). This would be especially useful if the EVM measurements are solid and the schedule does not permit full run of the test coverage in a nanosatellite project. We now investigate the fidelity of expressing SNR and the waveform quality factor $\rho$ in terms of EVM. The SNR in $\mathrm{dB}$ and EVM are related to as

$$
E V M=100 \sqrt{\left[\frac{1}{10}\right]^{S N R / 10}}
$$

While EVM relates the offset voltage of the measured constellation from the reference, $\rho$ is the correlation coefficient of the measured constellation spectrum power with the reference (Aparin, 2001). The $\rho$ compares the two signals as a \% fraction. To the same degree as the EVM, and in general and simpler terms, $\rho$ compositely paints a picture of errors in magnitude and phase, IQ errors, gain compression, spectral leakage and of the nonlinearities. We define $\rho$ as

$$
\rho=\frac{S N R}{1+S N R}
$$

and relate it to EVM as

$$
E V M=\sqrt{\frac{1}{\rho}-1}
$$

Typically, in the SNR measurements, the noise is assumed to be AWGN, which is a zero mean model (Moer et al., 2002) having unlimited but uni- 
form spectral density (white). Therefore the noise power is infinite. These characteristics do not distort the amplitude or the phase of the modulation precisely where the distortion occurs in the real world, as the frequency response is constantly flat and phase varies linearly for all frequencies. This overly simplified yet generally useful view of the channel is the basis of noise in the SNR figure of merit. Keeping foregoing in the view, the digital communication theory generally assumes that AWGN is the sole source of errors. The noise, however, can manifest as impulsive spurs of finite bandwidth that occur randomly or intermittently, thus, the total channel noise is additive and multiplicative, containing all types of spurious components (amplitude compression, harmonics, IMD, phase distortion etc.).

For minimum EVM, $S N R=1 / E V M^{2}$. Substitution of Eq. 2 leads to a more precise and augmented quantitation of SNR, called Modulation Error Ratio (MER). It is the ratio of the constellation power to constellation total noise or error, and hence, we expect MER to be even worse than SNR (Steer, 2010).

$$
M E R=10 \log _{10}\left[\frac{\sum_{i=1}^{N}\left(I_{i}^{2}+Q_{i}^{2}\right)}{\sum_{i=1}^{N}\left(\Delta I_{i}^{2}+\Delta Q_{i}^{2}\right)}\right]
$$

Since we derive MER from EVM, the reference is again the ideal constellation and MER (SNR) is inversely proportional to EVM. When the transmission is affected by AWGN alone, MER is equivalent to SNR. In Eqs. 3, 4 and 5 MER is written as SNR, a more familiar term, and the measurements are inclusive of all sorts of noise. The mathematical formulation of EVM contributions (Noël \& Brousse, 2011) and EVM based other metrics becomes complex when the distortion effects are severally factored in the aggregate EVM calculation, see (Georgiadis, 2004) for instance.

Fig. 7 illustrate the measured and the estimated metrics during the thermal cycle. The EVM in $\mathrm{dB}$ corresponding to EVM error in \% is plotted on the y2-axis on top graph. An rms EVM error of $6.25 \%$ contributes $-24 \mathrm{~dB}$ of in-band noise. The expected EVM and SNR agree well with the measured SNR and EVM respectively, while the expected EVM from the measured $\rho$ is $1-1.5 \%$ higher. The measured SNR is $0.6 \mathrm{~dB}-0.7 \mathrm{~dB}$ higher than the expected SNR when referred to $\rho$. Similarly, the expected and the measured EVM and $\rho$ are well consorted against each other, whereas expected $\rho$ from the measured SNR is around 3.5\% worse. The errors in EVM peak, EVM \%, SNR and $\rho$ show a linear trend. The waveform quality is further evidenced by the I signal quality, as it varies with time, in the eye opening of Fig. 8 at 


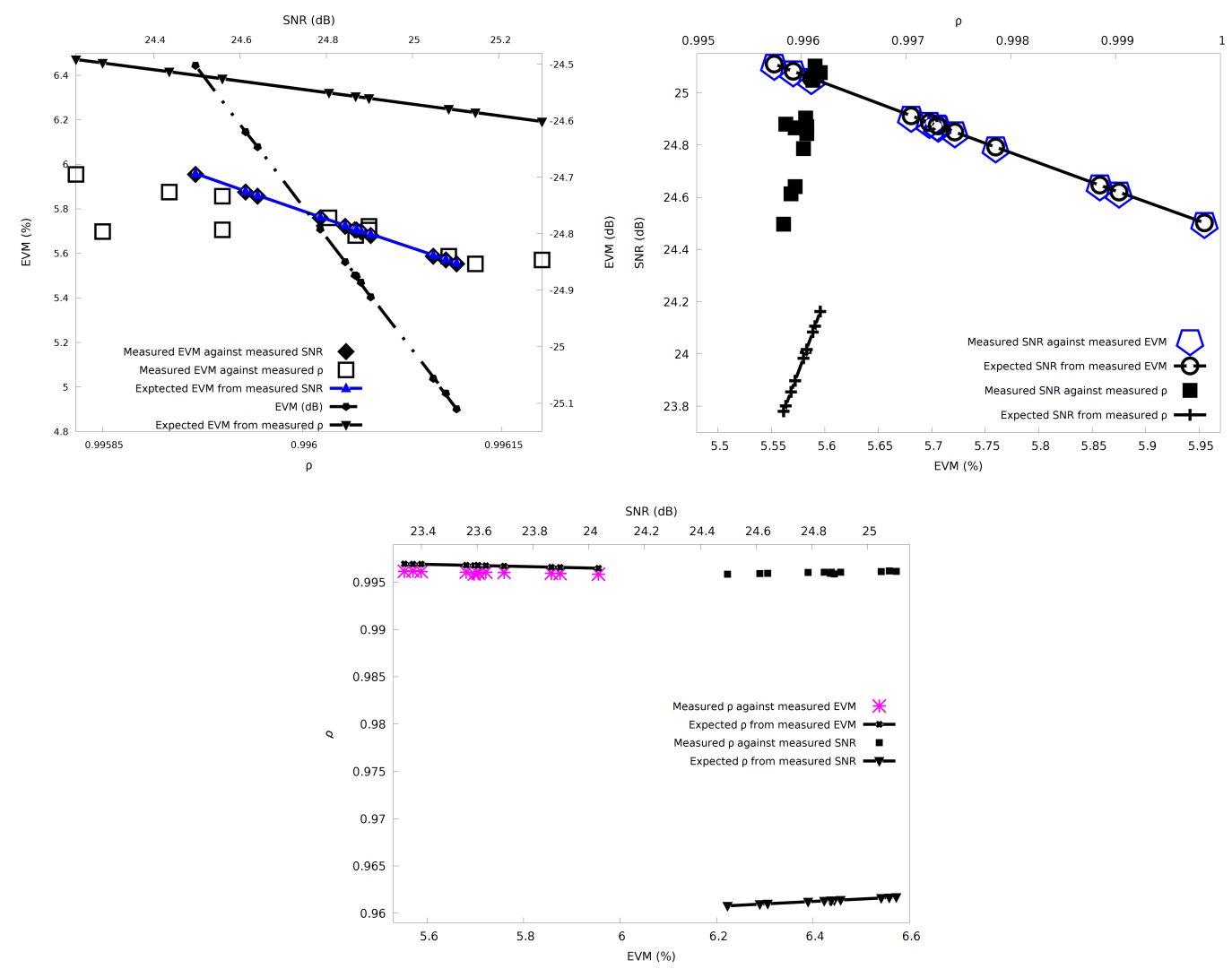

Figure 7: Measured and estimated EVM, SNR and $\rho$ at $24 \mathrm{dBm}$. 
$30 \mathrm{dBm}$ and the cycle extremes.
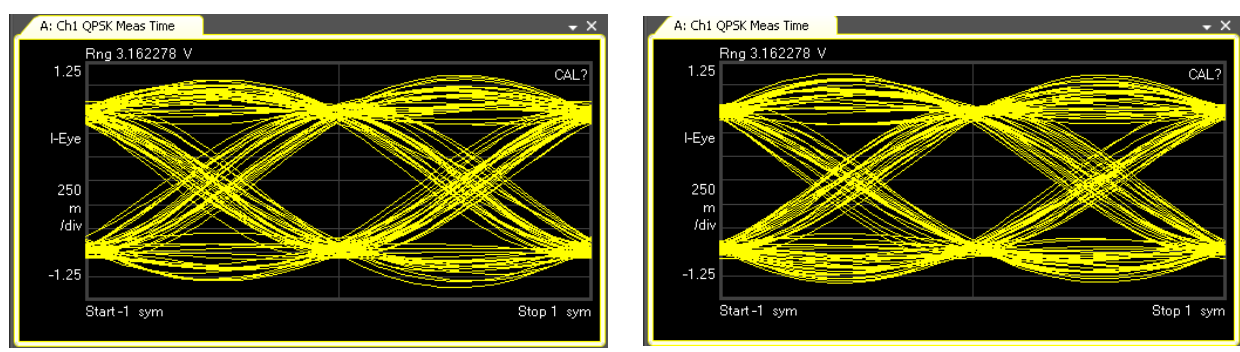

Figure 8: In-phase eye diagram at $30 \mathrm{dBm}\left(-19^{\circ} \mathrm{C}\right.$ left and $49.5^{\circ} \mathrm{C}$ right $)$.
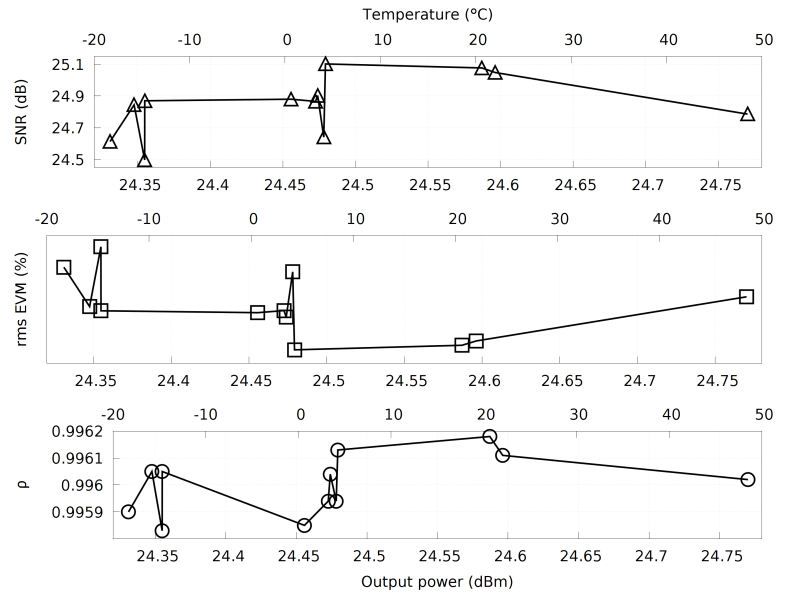

Figure 9: Relative change in SNR, EVM and $\rho$ with temperature at $24 \mathrm{dBm}$.

The top plot in Fig. 9 shows that during the thermal cycle from $5{ }^{\circ} \mathrm{C}$ to $20^{\circ} \mathrm{C}$ the $\mathrm{SNR}$ is the highest, the EVM error as expected is minimum in the middle plot, and $\rho$ is in excellent match in the bottom plot. The three metrics deteriorate toward the temperature extremes. Fig. 10 depicts, in surface and contours, the SNR against peak EVM variation. The peak EVM is dynamical as the measurement is made on a burst of symbols in $n$ timeslots, unlike the rms EVM which is measured at each timeslot.

\subsection{Gain Imbalance}

In a perfect channel the in-band gain is unity. Due to channel imperfections, the imbalance of gain is non-zero. The gain imbalance is computed 


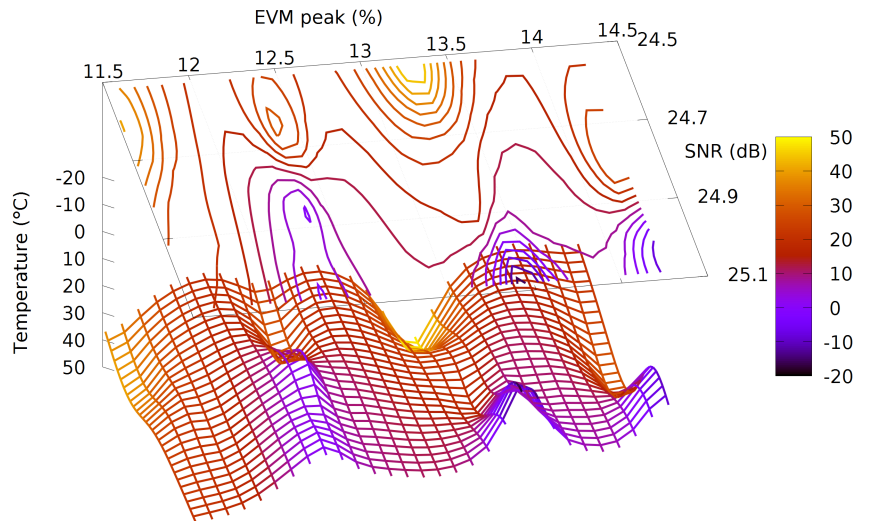

Figure 10: Surface and contour plot of EVM peak vs. SNR with variation in temperature at $24 \mathrm{dBm}$.

as

$$
\text { IQ Gain Imb. }=20 \log _{10}\left[\frac{I}{Q}\right]
$$
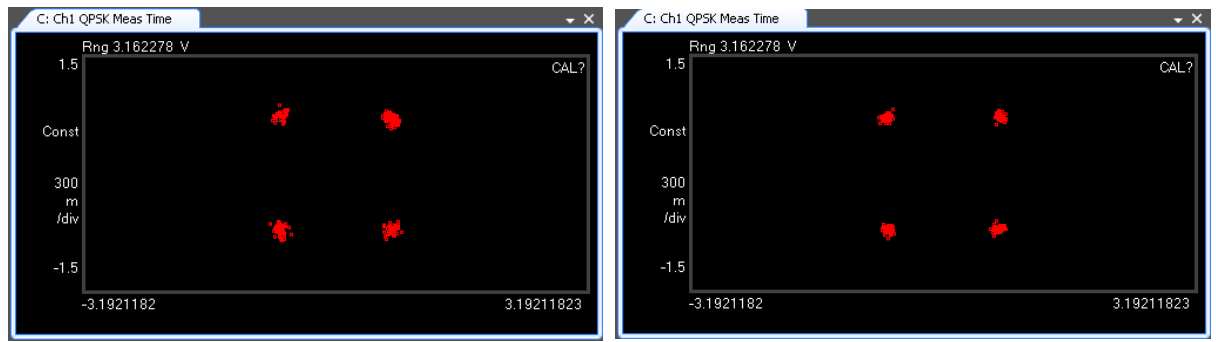

Figure 11: Constellation diagram at $30 \mathrm{dBm}\left(-19^{\circ} \mathrm{C}\right.$ left and $49.5^{\circ} \mathrm{C}$ right $)$.

The imbalance of gain is expected to exacerbate at higher power levels. Fig. 11 shows constellation diagram of four possible symbols at $30 \mathrm{dBm}$ level. The imbalance in I and Q gains is marginal at $-19^{\circ} \mathrm{C}$ and $49.5^{\circ} \mathrm{C}$ as the constellation width and height reasonably equalize. On both extremes, the I gain is slightly asymmetric and higher than the Q gain. Note also the tightness of the symbol cluster at each of the four locations; the errors are packed together and this means high SNR. Obviously, the transmitter is sensitive to very low temperatures, more than it is at the higher ones. At $-19^{\circ} \mathrm{C}$ the cluster is fuzzier and the rambling in the symbols is higher. The 
spread is in agreement with the rms EVM, $5.9 \%$ and $5.75 \%$, at $-19{ }^{\circ} \mathrm{C}$ and $+49^{\circ} \mathrm{C}$ respectively.

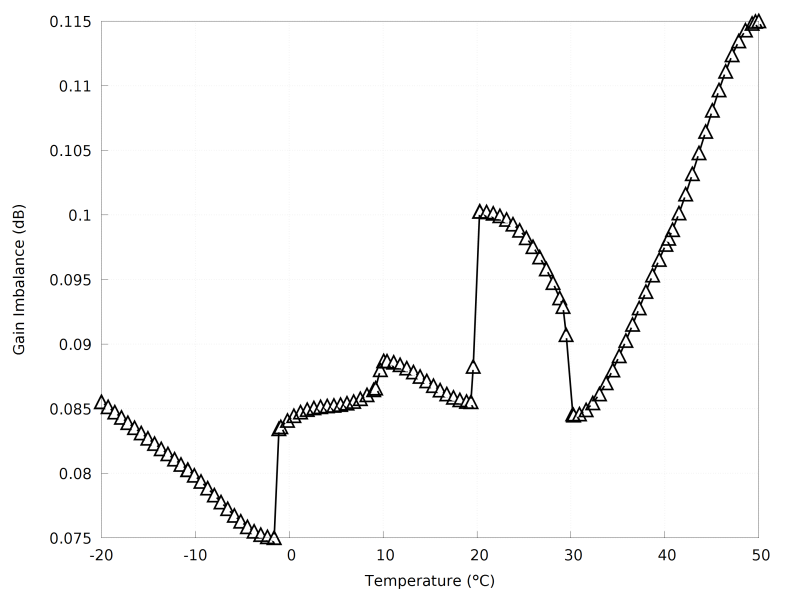

Figure 12: Imbalance in the I and Q gains during thermal cycle.

Fig. 12 shows the sensitivity of the variation in gain imbalance during the thermal cycle. The dynamic range of gain imbalance is $0.04 \mathrm{~dB}$ at $24 \mathrm{dBm}$ level during the temperature extremes. The jump at $20^{\circ} \mathrm{C}$ is due the two measurements taken, one at transitioning from hot to cold and the other from cold to hot.

The imbalance of IQ gain is related to EVM error. This effect is shown in Fig. 13. The surface of rms EVM is flatter and steadily inclined. Large IQ gain imbalance contributes to incorrect detection of the signal and the penalty is paid in high error rate.

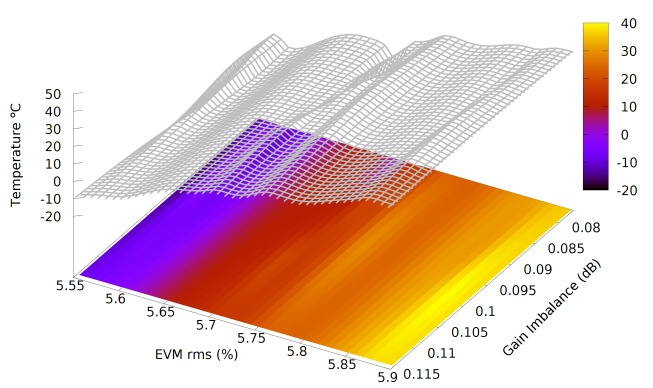

Figure 13: Surface plot of EVM rms vs. gain imbalance with variation in temperature at $24 \mathrm{dBm}$. 


\subsection{IQ Errors}

DC offset in either I or Q shifts the constellation from the origin. The source of the offset is amplitude level of unmodulated carrier feedthrough appearing with the modulated carrier at the receiver. The offset usually arises in the upconversion where the mixer $\mathrm{RF}$ and IF ports are not completely isolated and RF-to-IF and IF-to-RF couplings occur, largely the former as RF LO feedthrough. The mixer ports may be protected by filtering, especially with a BPF to attenuate $\mathrm{LO}$ feedthrough. Also, the error contribution is related to the phase noise and temperature dependent instability of the LO at the IQ mixers.
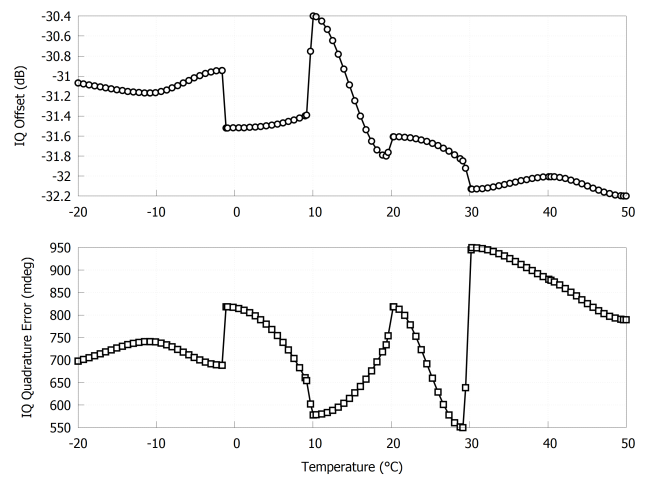

Figure 14: Errors in the IQ offset and quadrature in transmission at $24 \mathrm{dBm}$.

In Fig. 14, the maximum change in the $\mathrm{IQ}$ offset error between temperature extremes is $-1.8 \mathrm{~dB}$. Imperfect orthogonal quadrature phase multiplication by the mixer introduces error in phase imbalance. This effect appears in the constellation being a trapezoid i.e., the square becomes skewed clock or anticlockwise, depending on the sign of the phase error. No discernible tilt is seen in Fig. 11. In bottom illustration of Fig. 14, the phase error is held at $0.55^{\circ}$ to $0.95^{\circ}$ during the thermal cycle. This result from linear modulation is vital for the up coming power amplification in the signal processing path which typically tends to distort the linear phase. Between $30^{\circ} \mathrm{C}$ to $50{ }^{\circ} \mathrm{C}$, the offset is the best while the phase is the worst. Overall, the IQ offset and phase error indicate a piecewise linear channel response in each $10^{\circ} \mathrm{C}$ step and no perceptible indication of spurious, filtering ripples or nonlinear character can be caught. Fig. 15 illustrates the surface plot of errors in thermal cycle. 


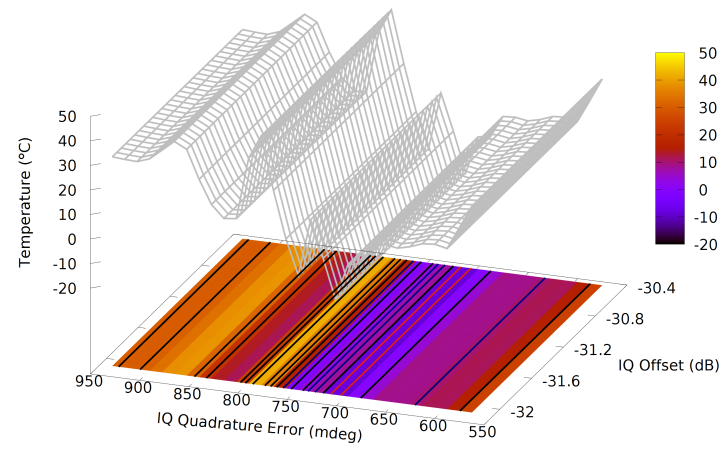

Figure 15: Surface plot of errors in the IQ quadrature and IQ offset during thermal cycle at $24 \mathrm{dBm}$.

\subsection{Magnitude, Phase and Frequency Errors}

A general problem in phase based modulations is the coherent detection at the receiver when the phase information is used during carrier recovery. A drift in carrier frequency outside of tolerance will create timing problems at the ground station to recover the symbol clock, and in correlating it with the reference signal/GPS. Similarly, faint magnitudes result in barely perceptible reception. All these problems result in excessive time in link acquisition during the satellite pass. Even if the RF link is established, its quality will be poor, contributed by the BER in downlinking.

In view of the geometric definition of the EVM resultant phasor, it is not possible to differentiate the errors in magnitude and phase component vectors severally. These are complementary errors, one will automatically result in the other, although one may dominant the other. To resolve this, errors in magnitude phase and frequency are separately measured. Ordinarily, the contributions are equatable, however, any imbalance gives insight of the channel distortion and the sensitivity of magnitude or the phase.

Fig. 16 relates magnitude, frequency and phase errors over the temperature range. Note the complementary effect in magnitude and phase in the top and middle plots. Fig. 17 relates the same effect in surface plot with contours. In the bottom plot of Fig. 16, the carrier frequency stability decreases with the rise in temperature while the fluctuation in frequency between the two extremes of temperature is a mere $-3600 \mathrm{~Hz}$ to $-1400 \mathrm{~Hz}$ which amounts to a maximum $0.163 \%$ error in carrier central frequency, and therefore, the stability of the LO over temperature is confirmed. Negative frequency errors indicate the tendency of constellation rotating clockwise. This observation 

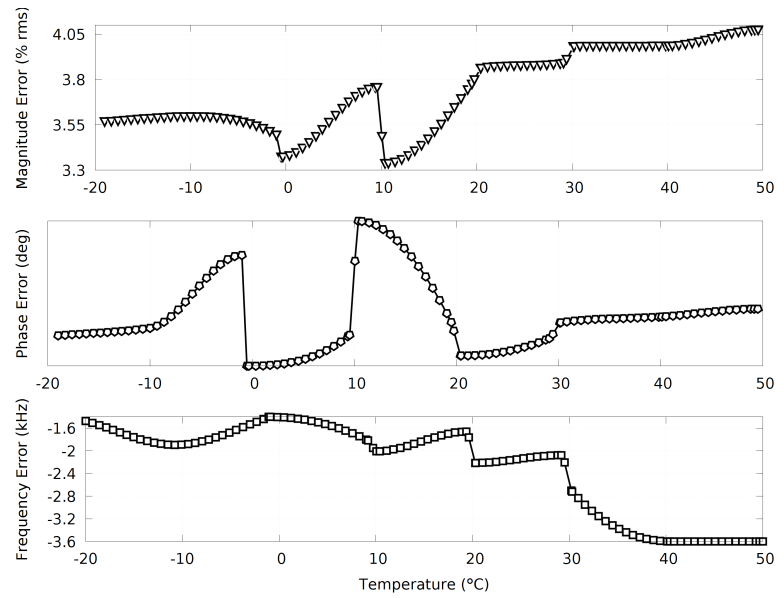

Figure 16: Relationship of errors in magnitude, phase and carrier frequency during thermal cycle at $24 \mathrm{dBm}$ output.
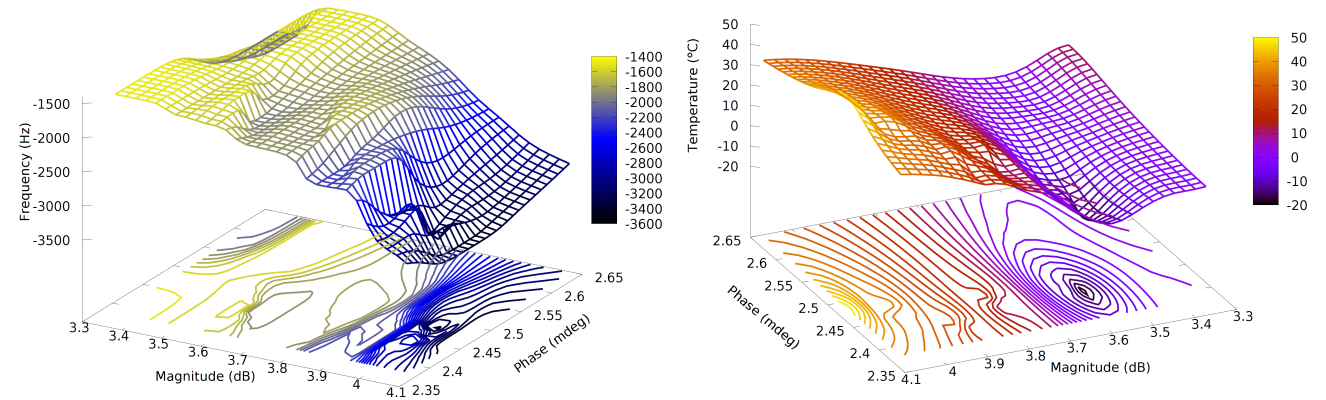

Figure 17: Surface and contour plot of errors in magnitude, phase and carrier frequency at $24 \mathrm{dBm}$ output in the thermal cycle.

is consistent with the gain imbalance measurement in Section 4.4, where the I gain is higher than $\mathrm{Q}$ gain.

From the surface plot, it is clear that the magnitude errors subdue with low frequency errors while large errors in the phase go along with large errors in frequency. The magnitude and phase errors show different sensitivity to temperatures. The magnitude rms error ranging $3.3 \%$ to $4.1 \%$ suggests a flatter frequency response i.e., absence of harmonic ripples or spurs (nonlinearity) such that the frequencies over the bandwidth are likely to be uniformly attenuated. On the whole, the magnitude error is somewhat larger than the phase error. The standard deviation in magnitude, phase, frequency and waveform quality are $0.6359 \mathrm{rms}, 0.4790 \mathrm{deg}, 95.8484 \mathrm{kHz}$ and 0.0088 respectively at 
$24 \mathrm{dBm}$ over the thermal cycle.

Having confirmed the modulation accuracy through a number of parameters, we can now, with reasonable confidence, divert to RF power amplification element of the transmission problem.

\section{Distortions Due to System Nonlinearities}

While stepping up the RF signal in strength, the PA must also track the variations in amplitude and phase of the input and amplify the signal with according proportions (class A operation). However, the nonlinearties in the amplifier distort AM-AM and AM-PM conversions. The nonlinear behavior manifests itself as appearance of RF power outside the in-band and the ensuing in-band becomes wider.

\subsection{Bias Control and Linear Amplification}

A linear PA can maintain the phase and amplitude proportionality from the input to the output if the amplitudes are small, a necessary requirement for low noise environment. Upon small changes in voltage and current amplitudes, proportionate linearity is ensured in the output by restricting the device operation to short segment on the $I-V$ characteristic loadline. An advantage of linear PAs is that different load impedances can be driven. This is especially useful in amplifying a wide range of frequency bands. The main disadvantage of a linear amplifier is low efficiency due to constant current draw at the output transistor even in the absence of RF input i.e., the conduction angle of a truly linear PA is $360^{\circ}$ and the driver current flows for the full period of the signal. To secure low noise and high gain in the output, an important aspect of linear PA operation is to bias it in the middle of $I-V$ transfer. When low or high variations in RF input occur, the PA compensates or swings the output on either side of the bias point. To be able to do this, the load and source impedances must have good match for good power transfer. On the contrary, in a fixed bias operation, some DC power is always wasted when no RF amplification is occurring. When the RF signal is smaller than the small-signal level, the amplifier still draws collector current. In the ideal world, for very small signals, the collector-to-emitter quiescent voltage $V_{C E Q}$ should come close to the supply voltage $V_{C C}$ and the quiescent collector current $I_{C Q}$ should approach zero. Therefore, efficiently shifting the bias point is an important requirement to accommodate a variety of input 
amplitudes. To proportionally amplify the amplitude variations, active biasing technique (Saleh \& Cox, 1983) is used in STX to bias the amplifier relative to the voltage of the envelope observed at the input.

\subsection{Harmonics and Intermodulation Distortion (IMD)}

Assume that the nonlinearity is such that the input is small and slow varying, then $x(t)=A \cos \omega_{c} t$ can be expanded as a finite order polynomial

$$
x_{n}(t)=\sum_{k=0}^{n} a_{k} x^{k}(t)
$$

where the power series coefficients $a_{k}$ are the positive or the negative homogeneity scaling factors. The 3rd order harmonically distorted signal is

$$
\begin{array}{r}
\widetilde{x_{3}(t)}=\left[\frac{A^{3} a_{3}}{4}\right] \cos 3 \omega_{c} t+\left[\frac{A^{2} a_{2}}{2}\right] \cos 2 \omega_{c} t \\
+\left[\frac{3 A^{3} a_{3}}{4}+A a_{1}\right] \cos \omega_{c} t+\left[\frac{A^{2} a_{2}}{2}+a_{0}\right]
\end{array}
$$

where the first two terms in [] are the linear gains of harmonics and $\left[\frac{3 A^{3} a_{3}}{4}+A a_{1}\right]$ is the gain of the fundamental. The coefficients $a_{k}$ depend on a number of semiconductor device level parameters. In reality, these values are very small for small-signal model (Grosch, 1999). We observe that the distortion in the amplitude power is related to the order of the harmonic. This is unwanted in QPSK detection which relies on the envelope amplitude from a small input. The 3rd, 5th and 7th harmonics are very similar to each other and the most troublesome. It follows from the power series that, in the AM-AM conversion, all higher order nonlinearities result in slopes equal to the order. For the cubic case, $1 \mathrm{~dB}$ variation in the input amounts to $3 \mathrm{~dB}$ in the output which is distorted by $2 \mathrm{~dB}$.

Nonlinearities in the mixer produces distortion as harmonics appearing in multiples of the fundamentals $\left(m f_{1}, n f_{2}\right)$ and intermodulating harmonics $\left(m f_{1} \pm n f_{2}\right)$. In much the same way as the harmonics, the 3rd order InterModulation Distortion (IMD3) $2 f_{1}+f_{2}, 2 f_{1}-f_{2}, 2 f_{2}+f_{1}, 2 f_{2}-f_{1}$ is the most devastating of these as the difference pair could occur in the vicinity of the fundamentals or even within the channel. 


\subsection{PA Nonlinearities - Gain Compression and Spectral Growth}

We note the fundamental's gain in Eq. 9 may have both positive and negative homogeneity in $a_{1}$ and $a_{3}$. In the 3 rd order case $x_{3}(t)=a_{0}+$ $a_{1} A \cos w_{c} t+a_{2} A^{2} \cos ^{2} w_{c} t+a_{3} A^{3} \cos ^{3} w_{c} t$, depending on their signs, the behavior of $a_{1}$ and $a_{3}$ in $\widehat{x_{3}(t)}$ may be suppressing or enlarging with respect to $a_{2}$. These combinations relate to the device's region of operation. It follows that the amplifier may not necessarily always amplify the input, rather, it might hold the gain flat or constrict it beyond certain limit. To avoid this nonlinearity, the operation of amplifier must be restricted to the all important linear region.

Besides gain compression, the most severe drawback of the PA nonlinearity is the spread of spectrum around the desired channel which defiles the QPSK philosophy of spectral efficiency. By adding two tones $\omega_{1}$ and $\omega_{2}$, the 3rd order static nonlinearity $\left(\cos \omega_{1} t+\cos \omega_{2} t\right)^{3}$ spectrally grows as

$$
\begin{array}{r}
\frac{3}{4}\left[\cos \left(2 \omega_{2}+\omega_{1}\right) t+\cos \left(2 \omega_{2}-\omega_{1}\right) t+2 \cos \omega_{1} t\right]+ \\
\frac{3}{4}\left[\cos \left(\omega_{2}+2 \omega_{1}\right) t+\cos \left(\omega_{2}-2 \omega_{1}\right) t+2 \cos \omega_{2} t\right]+ \\
\frac{1}{4}\left[\cos 3 \omega_{2} t+3 \cos \omega_{2} t+\cos 3 \omega_{1} t+3 \cos \omega_{1} t\right]
\end{array}
$$

Clearly, the RF power may leak to the spectra close to the in-band. The channel spacing in STX is $500 \mathrm{kHz}$, in steps of which, the carrier can be adjusted within the band.

\subsection{Amplifier Performance and Nonlinear Analysis}

In the contradicting goals of system linearity and RF power conversion efficiency, linearity takes precedence whether the transmission is service based or of a nanosatellite. The PA efficiency becomes important in view of the small mass and surface area of the nanosatellites and the modes of heat transfer, because, the amount of energy wasted appears as resident heat. The standing heat waves between two cards in the satellite structure or their reflections from neighboring cards to the STX may adversely affects not just the communication subsystem performance but that of the whole satellite. We now look into just how far out the PA operation goes in the linear region and what efficiency can be expected at different output power levels. 


\subsection{Trade-offs in RF Power, Modulation Quality, DC Biasing and Temper- ature}

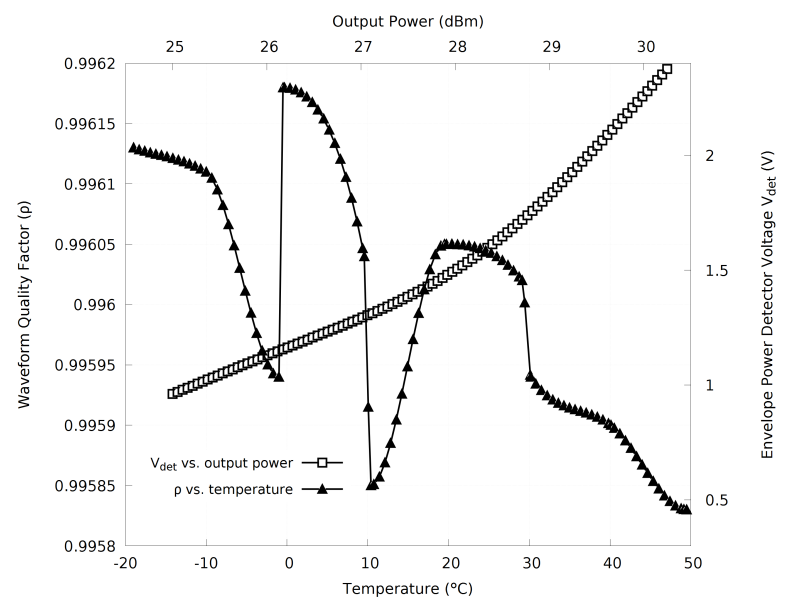

Figure 18: Behavior of waveform quality factor and variation in amplitude at the power detector as output RF power is stepped up from $24 \mathrm{dBm}$ to $30 \mathrm{dBm}$ during the thermal cycle.

In the STX the RF envelope level is proportionally returned at the power detector of the amplifier. Fig. 18 depicts the detector voltage and the output waveform quality factor as both vary with the temperature and the output power respectively. The voltage rises steadily from $1 \mathrm{~V}$ to $2.5 \mathrm{~V}$ for $28 \mathrm{dBm}$ to $30 \mathrm{dBm}$ power sweep respectively. As the temperature increases the waveform quality declines at higher power levels. The best transmission performance is at $26 \mathrm{dBm}$ to $27 \mathrm{dBm}$ output related to a temperature field of $0{ }^{\circ} \mathrm{C}$ to $10^{\circ} \mathrm{C}$. Another good window of operation is $-20^{\circ} \mathrm{C}$ to $0{ }^{\circ} \mathrm{C}$, however, the detector voltage is about $1 \mathrm{~V}$, which corresponds to moderate RF output $(24.5 \mathrm{dBm}$ to $26 \mathrm{dBm})$. In all likely ConOp, the trajectory will be in the eclipse. The operation will be battery powered. As a general rule in spacecraft operations, the battery is usually conserved for any active operation during the unavailability of sun. A situation in which battery may be used for cryogenic transmission is in the early life of the satellite when the rated capacity of the battery is the highest and the depth of discharge is significantly high (90\% or more). It must be noted that for any chemical composition of the spacecraft batteries, as well as the Li polymer technology typically flown in the nanosatellites, battery's depth of discharge is a strong function of ambient temperature. The transmission at very low temperatures 
should be avoided, unless critical health monitoring data is required to be downloaded. A probable operation scenario is $25^{\circ} \mathrm{C}$ to $50^{\circ} \mathrm{C}$ range, which is apparent sunlit condition and thus the electric power off the solar panels will be available for the PA to operate at the highest level. The downside of this use case would be as more and more RF power is pumped out $(28.5 \mathrm{dBm}$ to $30 \mathrm{dBm}$ ), the temperature gradient will be lower for heat to escape from the spacecraft structure to the outer space.

In Fig. 18 the waveform quality is high throughout the cycle i.e., more than $99.58 \%$ correlated power is contained in-band and the carrier leakage to the adjacency is a mere $0.4 \%$. This is higher than typical specifications of the terrestrial radio link services.

At $30 \mathrm{dBm}$, the ConOp is suitable for missions at higher altitudes in the low earth orbit regime where higher output power may be required. Another advantage of transmission at $30 \mathrm{dBm}$ is in the antenna pattern and directivity at the ground segment, if not optimum, the higher power level from the space segment will support the mismatch on the ground.

High RF power transmission at high temperature may be supported by thermal cycle or balance test of the integrated spacecraft or, at the very least, with the FE thermal model incorporating realistic thermal margins. FE simulation coupled with orbit and mission related thermal data has added advantage over standard FE codes as some FE tools use altitude, launch epoch and solar cycle information, as well as orbital heating and thermalvacuum pressure models to predict the in-orbit temperature fields.

\subsection{Efficiency}

Fig. 19 illustrates the RF-to-DC efficiency of the amplifier in power sweep over thermal cycle. The efficiency profile is fairly linear for lowest power transmit level. At higher RF level the efficiency is better, as expected. In $30 \mathrm{dBm}$ mode, the variation in efficiency is due to the minor nonlinearities than the thermal instability. Overall the efficiency is no better than $30 \%$. This is the high price of linearity.

\section{7. $1 \mathrm{~dB}$ Compression Point}

Fig. 20 shows the amplifier backoff margin extrapolated from the manufacturer specified $1 \mathrm{~dB}$ compression point at which the output is $1 \mathrm{~dB}$ less than the hypothetical, consummate linear, distortion-free case, only possible if the gain were continue to increase. The point is the end limit of linearity and the beginning of nonlinearity, at which the monotonic amplification 


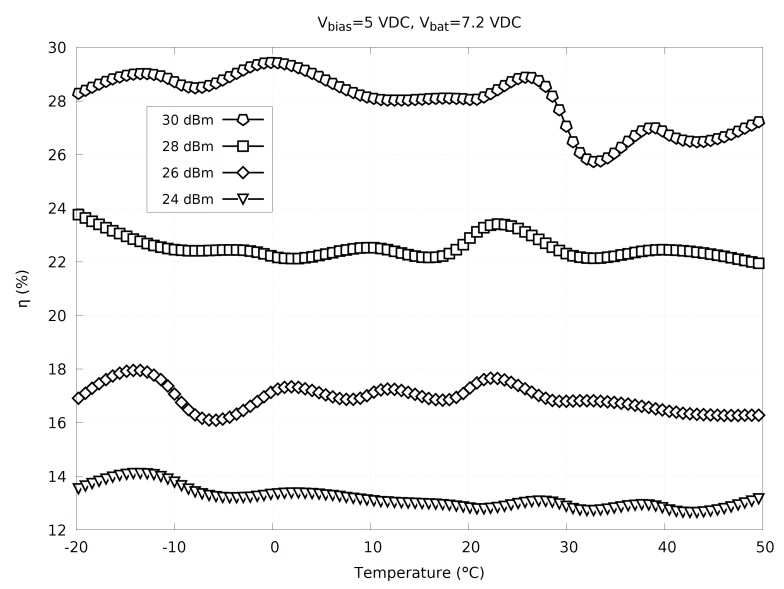

Figure 19: Power amplifier efficiency during thermal cycle at $2200 \mathrm{MHz}$ when the bias is of a DC-to-DC converter connected to a fully regulated battery bus.

ceases and any increase in the input power would level out to flat in the output or even the gain constricts. Since the RF conversion does not increase, any more input power is wasted as heat. The peak output power possible of the amplifier occurs at $1 \mathrm{~dB}$ point. At the maximum output power of $30 \mathrm{dBm}$, in Fig. 20, the power allowances comfortably exceed $1 \mathrm{dBm}$ in $25^{\circ} \mathrm{C}$ to $50^{\circ} \mathrm{C}$ window. In terms of efficiency, the compression region is $0.36 \mathrm{~W}$ to $0.5 \mathrm{~W}$ away from the $30 \mathrm{dBm}$ operation. The backing off is in accordance with Eq. 9 to secure a linear relationship with the DC component.

Although as negligible as it may seem, to attain an additional $\mathrm{dBm}$ in the output and considering the limitations in the efficiency, the amplifier must be driven extremely hard. Even if ever reached, the transmission performance will badly deteriorate beyond the compression point as the collector current amplitudes contributed by the higher order terms in the power series eventually become more powerful.

\subsection{Third-Order Intercept Point (IP3)}

The Third order Intercept Point (IP3) is an extrapolation if the inputoutput linearity and pro rata of the gain were to hold beyond $1 \mathrm{~dB}$ compression. IP3 is where the third order Input Intercept Point (IIP3) and Output Intercept Point (OIP3) intercept each other. At IIP3/OIP3 the amplitudes (power) of the in-band input/output intermodulation tones $2 f_{1}-f_{2}$ and $2 f_{2}-f_{1}$ theoretically equalize their input/output fundamentals $f_{1}$ and $f_{2}$ 


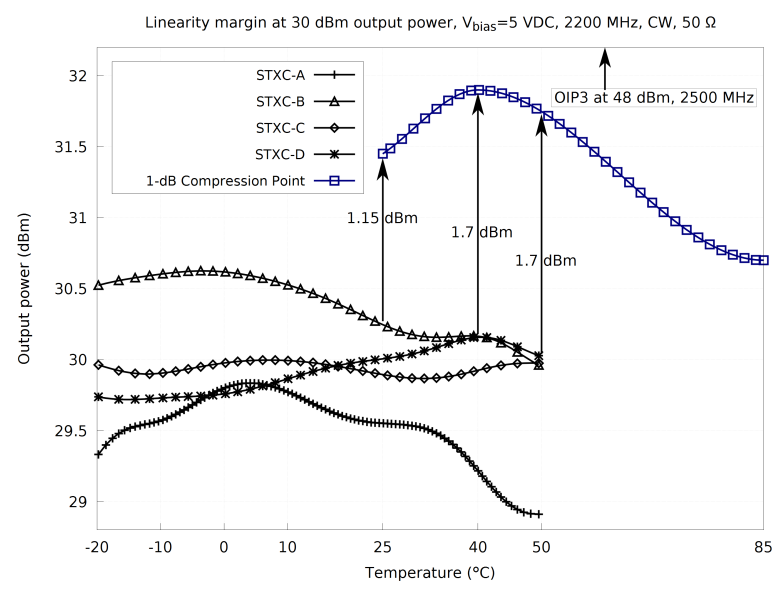

Figure 20: At maximum output of $30 \mathrm{dBm}$ the power margin to the anticipated $1 \mathrm{~dB}$ compression point for multiple STXC cards is very respectable $(1.15 \mathrm{dBm}-1.7 \mathrm{dBm})$ during the entire thermal cycle.

(Rogers \& Plett, 2003). A first-order estimate of IIP3 and OIP3 can be related as (Bahl, 2009)

$$
\begin{aligned}
& I I P 3=1 \mathrm{~dB}+10.6 \\
& O I P 3=G+I I P 3
\end{aligned}
$$

where $G$ is small-signal gain. The OIP3 is always higher than the IIP3 by an amount of linear gain and OIP3 will be at least $10 \mathrm{~dB}$ above $1 \mathrm{~dB}$ compression point.

IIP3 is the ultimate metric for quantifying a system for nonlinearity as it reveals the degree by which two close frequency tones intermix and distort the device's linear performance. In effect, IIP3 shows the margin between the input drive at the onset of distortion to a level at which the IMD3 distortion would have as much power spectral density as its fundamentals when amplified by the PA. For a $-5 \mathrm{dBm}$ single tone in a system with IP3 at $30 \mathrm{dBm}$, the margin to the dominant 3rd order distortion will be $45 \mathrm{~dB}$.

In Fig. 20 the OIP3 is expected to be somewhat further out as the carrier frequency is lower than $2500 \mathrm{MHz}$. This is a reasonably safe margin to the extreme nonlinearity, even at the maximum output RF power. 


\subsection{Occupied Channel Bandwidth (OCBW) and Adjacent Channel Power $(A C P)$}

Abrupt variation in phase with respect to time results in amplitude variation and the expense of more bandwidth (Scott \& Frobenius, 2008). In fact, any distortion in the signal path will appear in the out-of-band and hence the ever indispensable need of keeping the linearity throughout the path.

The reasons for the spectrum outgrowth are diverse and numerous - they include obtuseness in the roll-off during shaping the QPSK pulses, system harmonics, IQ imbalances, mixer IMD, PA nonlinearity, impedance mismatch and imperfections of filtering in removing out-of-band frequencies, to name a few. PA nonlinearities are the dominant contribution (Sayre, 2008; Aparin, 2001; Steer, 2010), recall Section 5.3. It is practically impossible to determine the number of significant harmonics carrying spectral component. The algorithm proposed in Moer et al. (2002) uses a stochastic technique and shows calculations up to the 41th harmonic of the amplifier input and a total 10 significant odd components. Instead, let's focus on the energy content of spectral regrowth.

The bandwidth occupied by the signal is given by Carson's rule $O C B W=$ $2 f_{\delta}+2 f_{m}$, where $f_{\delta}$ is the maximum deviation in the carrier's frequency and $f_{m}$ is the maximum frequency of the modulating signal. The significance of the given occupancy is that the energy contents as little as $1 \%$ of the main carrier amplitude are captured. We define the OCBW as a slice of the total, theoretically infinite bandwidth, in which any type of signal components whether fundamental, harmonics or inharmonics (Josephson, 1970) inhabit, and that contains $99 \%$ of the total integrated mean power in the transmitted channel.
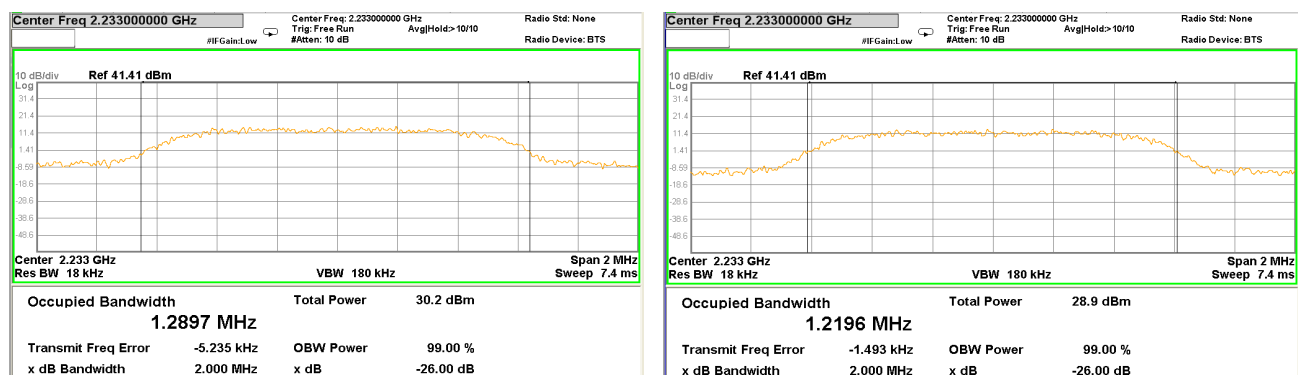

Figure 21: Occupied bandwidth at $30 \mathrm{dBm}\left(-19^{\circ} \mathrm{C}\right.$ left and $49.5^{\circ} \mathrm{C}$ right $)$.

Fig. 21 depicts the spectral dispersal in the worst case conditions. The 
transmitter is more sensitive at the lower temperatures, the frequency error increases and so does the OCBW. Though negligible, the dispersal is $70 \mathrm{kHz}$ broader at $-19^{\circ} \mathrm{C}$ than at $+49^{\circ} \mathrm{C}$.

Virtually, all of the spectral energy is trammeled in the main channel and the two side lobes, the upper and lower adjacent channels, just above and below the fundamental. The power of the two contiguous channels is the ACP figure of merit.
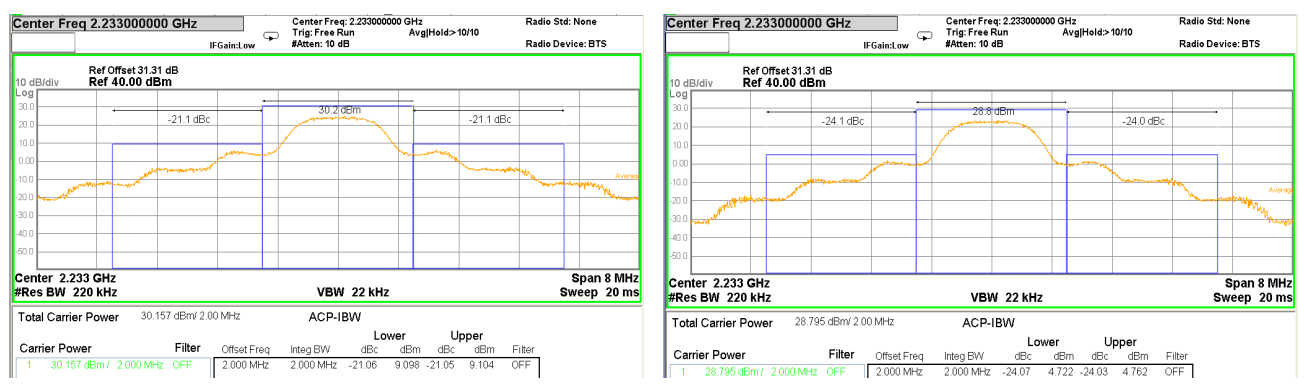

Figure 22: Spillage of power in side bands is rarefied at $30 \mathrm{dBm}\left(-19^{\circ} \mathrm{C}\right.$ left and $49.5^{\circ} \mathrm{C}$ right).

In Fig. 22 at maximum power level $30.2 \mathrm{dBm}$, the ACP in the upper and lower side lobes is about $9.1 \mathrm{dBm}$ at $-19^{\circ} \mathrm{C}$, and about $4.7 \mathrm{dBm}$ at $49.5^{\circ} \mathrm{C}$. The Adjacent Channel Power Ratio (ACPR) is the ratio of power in one side lobe to the channel power. We define it as (Roblin, 2011)

$$
A C P R=10 \log _{10}\left[\frac{\int_{\omega_{\text {adjacent }}} P S D(\omega) d \omega}{\int_{\omega_{\text {channel }}} P S D(\omega) d \omega}\right]
$$

This leads to ACPR of $-21.1 \mathrm{dBc}$ for each side lobe at $-19^{\circ} \mathrm{C}$ and, $-24 \mathrm{dBc}$ in each side lobe for $+49^{\circ} \mathrm{C}$ case. Note that outside of the integrated bandwidth $(2.0 \mathrm{MHz})$ the signal level is about $-12 \mathrm{~dB}$ and $-20 \mathrm{~dB}$ or below at $-19^{\circ} \mathrm{C}$ and $+49^{\circ} \mathrm{C}$ respectively. The offset frequencies used were $\pm 2.0 \mathrm{MHz}$, while the radio standard is set to none as no formal compliance to a particular radio transmission is necessary and the radio device applied is the base transceiver station. This measurement is the most stressful both from temperature and power standpoints and also from performance aspect as the rrc filter is disabled on the analyzer due to the radio standard compliance being switched off.

The ACP progressively increases with hike in RF power. This can be seen as the size of surfaces in Fig. 23. Also, the corresponding contour plots are 

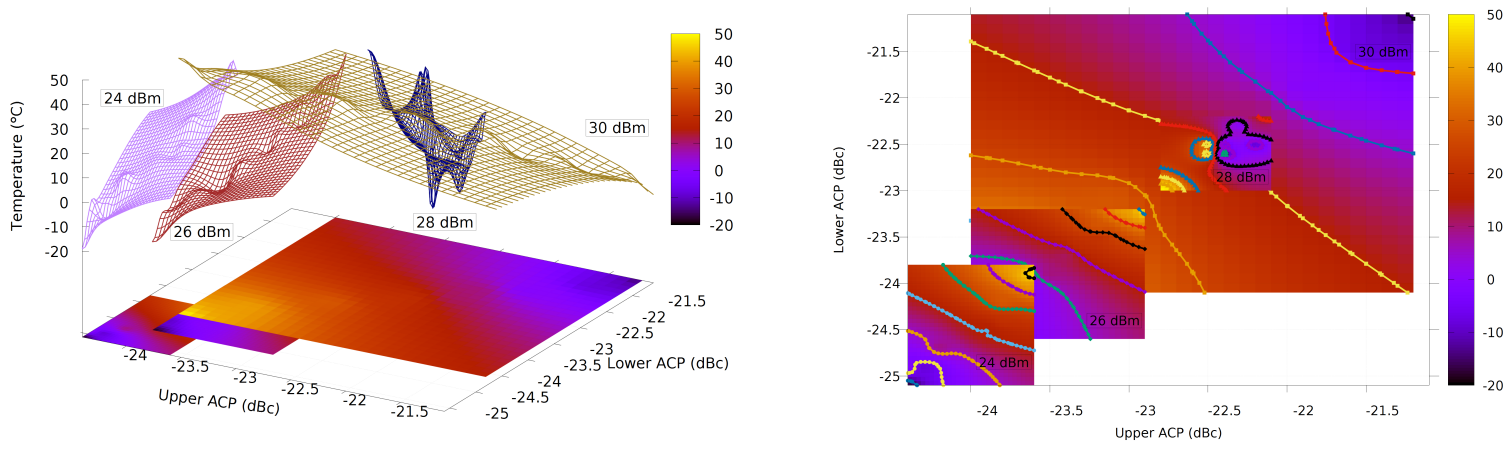

Figure 23: Surface and contour plots of power leakage to upper and lower channels at $24 \mathrm{dBm}, 26 \mathrm{dBm}, 28 \mathrm{dBm}$ and $30 \mathrm{dBm}$ outputs during thermal cycle.

tighter for low powers. Similar relative effects of OCBW spread on the ACP can be seen in Fig. 24.

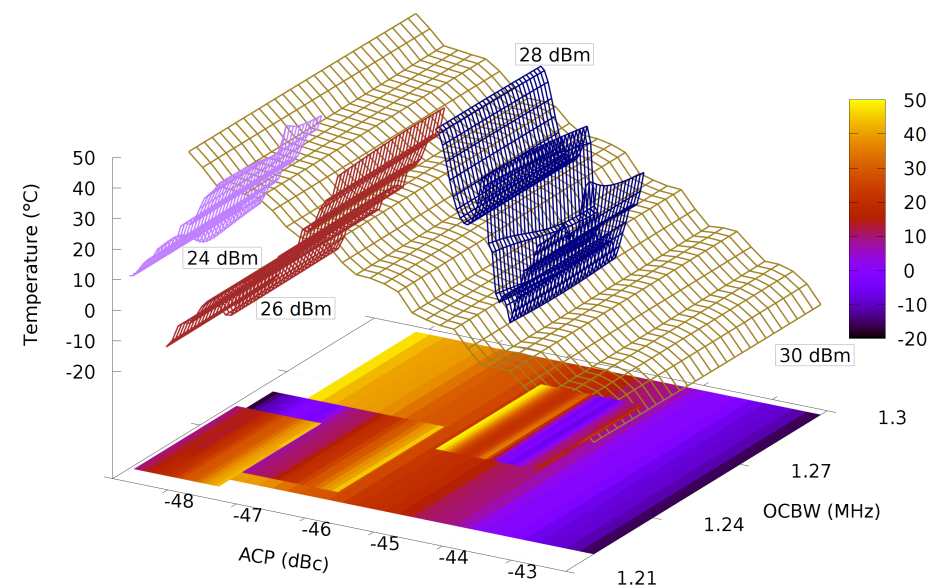

Figure 24: Relating adjacent channel power to the occupied bandwidth at $24 \mathrm{dBm}, 26 \mathrm{dBm}, 28 \mathrm{dBm}$ and $30 \mathrm{dBm}$ outputs during thermal cycle.

One of the reasons of OCBW spread and ACP is the carrier frequency jitter in the channel. Fig. 25 shows the distribution of central frequency errors as heights in the histogram. The frequency error in part is related to the difference in channel central frequency and the measurement testset frequency. The maximum errors in frequencies in the whole thermal cycle are $4590 \mathrm{~Hz}$ and $-7454 \mathrm{~Hz}$. By Carson's rule, the contributions of the two deviations on widening of their occupied bandwidths $(1.246 \mathrm{MHz}$ and $1.2677 \mathrm{MHz})$ are $0.74 \%$ and $1.18 \%$ respectively. This result is negligible. Bandwidth moder- 
Table 1: Summary of Spectral Growth and Adjacent Channel Power for 99\% of the Bandwidth Occupancy in Thermal Cycle

\begin{tabular}{|c|c|c|c|c|c|c|c|c|c|c|c|c|c|c|c|c|c|c|c|c|}
\hline \multirow[b]{2}{*}{$\begin{array}{l}\text { Temp. } \\
\left({ }^{\circ} \mathrm{C}\right)\end{array}$} & \multicolumn{5}{|c|}{$24 \mathrm{dBm}$} & \multicolumn{5}{|c|}{$26 \mathrm{dBm}$} & \multicolumn{5}{|c|}{$28 \mathrm{dBm}$} & \multicolumn{5}{|c|}{$30 \mathrm{dBm}$} \\
\hline & \begin{tabular}{|c|} 
Main \\
$\mathrm{Ch}$. \\
$(\mathrm{dBm})$ \\
\end{tabular} & \multicolumn{2}{|c|}{$\begin{array}{c}\mathrm{ACP} \\
(\mathrm{dBc}) \\
\text { Upper Lower }\end{array}$} & \multirow{2}{*}{$\begin{array}{c}\begin{array}{c}\text { OCBW } \\
(\mathrm{MHz})\end{array} \\
1.2176\end{array}$} & \multirow{2}{*}{\begin{tabular}{|c|}
$\begin{array}{c}\text { Freq. } \\
\text { Err. } \\
(\mathrm{kHz})\end{array}$ \\
-1.474 \\
\end{tabular}} & \multirow{2}{*}{$\begin{array}{c}\begin{array}{c}\text { Main } \\
\text { Ch. } \\
(\mathrm{dBm})\end{array} \\
25.9\end{array}$} & \multicolumn{2}{|c|}{\begin{tabular}{|c|}
$\mathrm{ACP}$ \\
$(\mathrm{dBc})$ \\
Upper Lower
\end{tabular}} & \multirow{2}{*}{\begin{tabular}{|c|}
$\begin{array}{c}\text { OCBW } \\
(\mathrm{MHz})\end{array}$ \\
1.2237
\end{tabular}} & \multirow{2}{*}{\begin{tabular}{|c|}
$\begin{array}{c}\text { Freq. } \\
\text { Err. } \\
(\mathrm{kHz})\end{array}$ \\
0.552
\end{tabular}} & \multirow{2}{*}{$\begin{array}{c}\begin{array}{c}\text { Main } \\
\text { Ch. } \\
(\mathrm{dBm})\end{array} \\
28.0\end{array}$} & \multicolumn{2}{|c|}{$\begin{array}{c}\mathrm{ACP} \\
(\mathrm{dBc}) \\
\text { Upper Lower }\end{array}$} & \multirow{2}{*}{$\begin{array}{c}\begin{array}{c}\text { OCBW } \\
(\mathrm{MHz})\end{array} \\
1.2682\end{array}$} & \multirow{2}{*}{$\begin{array}{c}\begin{array}{c}\text { Freq. } \\
\text { Err. } \\
(\mathrm{kHz})\end{array} \\
0.041\end{array}$} & \multirow{2}{*}{\begin{tabular}{|c|}
$\begin{array}{c}\text { Main } \\
\text { Ch. } \\
(\mathrm{dBm})\end{array}$ \\
30.2
\end{tabular}} & \multicolumn{2}{|c|}{\begin{tabular}{|c|}
$\mathrm{ACP}$ \\
$(\mathrm{dBc})$ \\
Upper Lower
\end{tabular}} & \multirow{2}{*}{\begin{tabular}{|c}
$\begin{array}{c}\text { OCBW } \\
(\mathrm{MHz})\end{array}$ \\
1.2897
\end{tabular}} & \multirow{2}{*}{\begin{tabular}{|c|}
$\begin{array}{c}\text { Freq. } \\
\text { Err. } \\
(\mathrm{kHz})\end{array}$ \\
-5.235 \\
\end{tabular}} \\
\hline-19 & 24.5 & \begin{tabular}{|l|}
-24.4 \\
\end{tabular} & -25.1 & & & & \begin{tabular}{|l|} 
\\
\end{tabular} & $\begin{array}{l}-24.6 \\
\end{array}$ & & & & -22.4 & \begin{tabular}{|l|}
-22.6 \\
\end{tabular} & & & & -21.1 & -21.1 & & \\
\hline-10 & 24.5 & & & 12177 & 0.364 & 25.9 & -24 & & 1.2323 & 1.82 & 28.0 & & & 1.2677 & -7.454 & 30.2 & -21.3 & & 1.2978 & -4.655 \\
\hline-8.6 & 24.5 & 24.3 & -24 & 1.22 & -1.367 & 25.87 & 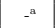 & $-^{\mathrm{a}}$ & 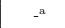 & $-^{\mathrm{a}}$ & 28.0 & -22.4 & -22 & 65 & -0.54 & 30.1 & -21.5 & -21.5 & 76 & 0.774 \\
\hline-1 & 2 & $\mid-24.3$ & -24 & 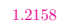 & -3.537 & 25.9 & -23.7 & -24.0 & .2360 & -6.1 & 28.0 & -22.1 & -2 & 16 & -1.052 & .0 & -21.9 & & 61 & 0.725 \\
\hline-0.5 & 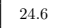 & 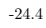 & -24 & & 0.95 & 25.9 & & & & $\mid-1.667$ & 28.0 & -22 & -2 & & 0.4 & .0 & -21.7 & & & -0.324 \\
\hline 10.4 & & -2 & -2 & & -3.2 & & & & & 0.8 & 0 & -20 & & & -3.795 & & -22.3 & & & 0.83 \\
\hline 9.56 & 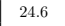 & -2 & -24 & & 1.0 & 0.3 & -23 & -2 & & $\mid-2.2$ & 27.9 & -22 & -2 & & -0.5 & $2 !$ & -22.2 & & 05 & -1.215 \\
\hline 19.5 & & -2 & -24 & & -2.5 & 25.9 & -23 & -2 & & 4.5 & 27.9 & -2 & & & 0.5 & & -22.6 & & & 1.347 \\
\hline 20.4 & 2 & -23 & -24 & 1.22 & -2.3 & 25. & -23.2 & -2 & 1.2456 & $\mid-5.3$ & 27 & -2 & -2 & 1. & -1.2 & 29.5 & -22.7 & -2 & 09 & -2.885 \\
\hline 29.1 & 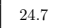 & -23 & -24 & & -3.9 & , & -23.2 & -2 & & -3. & 28 & -22 & -2 & & -0.7 & 2 & .1 & & & 2.170 \\
\hline 39.5 & 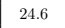 & -23 & -2 & & -4 & 25. & -23 & -2 & & & 27 & -2 & & & -5 & & & & 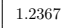 & -3.394 \\
\hline 40.1 & 24.5 & -2 & -23 & & 1.94 & 25.8 & $\mid-23.0$ & -2 & & $\mid-6.2$ & $2 \pi$. & -22 & -2 & 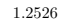 & -2.6 & 29 & -23.7 & -2 & & -1.906 \\
\hline 49.5 & 24.5 & $\mid-23.6$ & -23.9 & 1.239 & -0.954 & 25.8 & -22.9 & -23.2 & 1.2497 & $\mid-5.649$ & 27.8 & -22.8 & -23.0 & 1.2502 & \begin{tabular}{|l|}
-5.106 \\
\end{tabular} & 28.8 & -24.0 & -24.1 & 1.2196 & -1.493 \\
\hline
\end{tabular}

${ }^{\text {a }}$ No measurement due to Agilent Signal Analyzer VSA software glitch.

ately decreases at higher temperatures. The corresponding modulating waves are in the order of $618 \mathrm{kHz}-641 \mathrm{kHz}$. The errors were recorded at $+19.5^{\circ} \mathrm{C}$ and $-10^{\circ} \mathrm{C}$ respectively. The bandwidth occupancy is the lowest from $20^{\circ} \mathrm{C}$ to $30^{\circ} \mathrm{C}$ between $0 \mathrm{~Hz}$ to $1000 \mathrm{~Hz}$. Both ACP and OCBW tend to be lower for positive errors which exposes the inertial nature of the IQ constellation to counter the clockwise rotation.

Table 1 lists a summary of spectrum spread for power sweep and temperatures. We can make the following observations. Both channels are fairly symmetric, and thus, the presence of memory effects in the PA is ruled out (Zhou \& Raich, 2004). The lower channel tends to be slightly worse than the upper channel. This trend is related to the frequency error which is predominantly negative for the power sweep. The maximum difference in positive and negative frequency errors is $12 \mathrm{kHz}$ in all power levels and temperatures. The maximum level difference between the channels at each $24 \mathrm{dBm}$, $26 \mathrm{dBm}, 28 \mathrm{dBm}$ and $30 \mathrm{dBm}$ is $0.7 \mathrm{dBc}, 0.7 \mathrm{dBc}, 0.3 \mathrm{dBc}$ and $0.2 \mathrm{dBc}$, respectively. Thus, the channels are more balance in level at the higher outputs. The maximum variation in OCBW for full sweep and temperature range is no more than $82 \mathrm{kHz}$ or $(0.067 \%)$, indicated by the colored entries in Table 1. The standard deviation in OCBW is $0.0112 \mathrm{MHz}, 0.0116 \mathrm{MHz}, 0.0107 \mathrm{MHz}$ and $0.0263 \mathrm{MHz}$ and in frequency error $2.2590 \mathrm{kHz}, 3.9512 \mathrm{kHz}, 2.7396 \mathrm{kHz}$ and $2.5089 \mathrm{kHz}$ at respective $24 \mathrm{dBm}, 26 \mathrm{dBm}, 28 \mathrm{dBm}$ and $30 \mathrm{dBm}$ over the thermal cycle. As a last remark, the data fidelity of Table 1 shows that the repeatability problem associated with integration effect of the spectral density in power measurements (Angrisani et al., 2006) does not exist. 


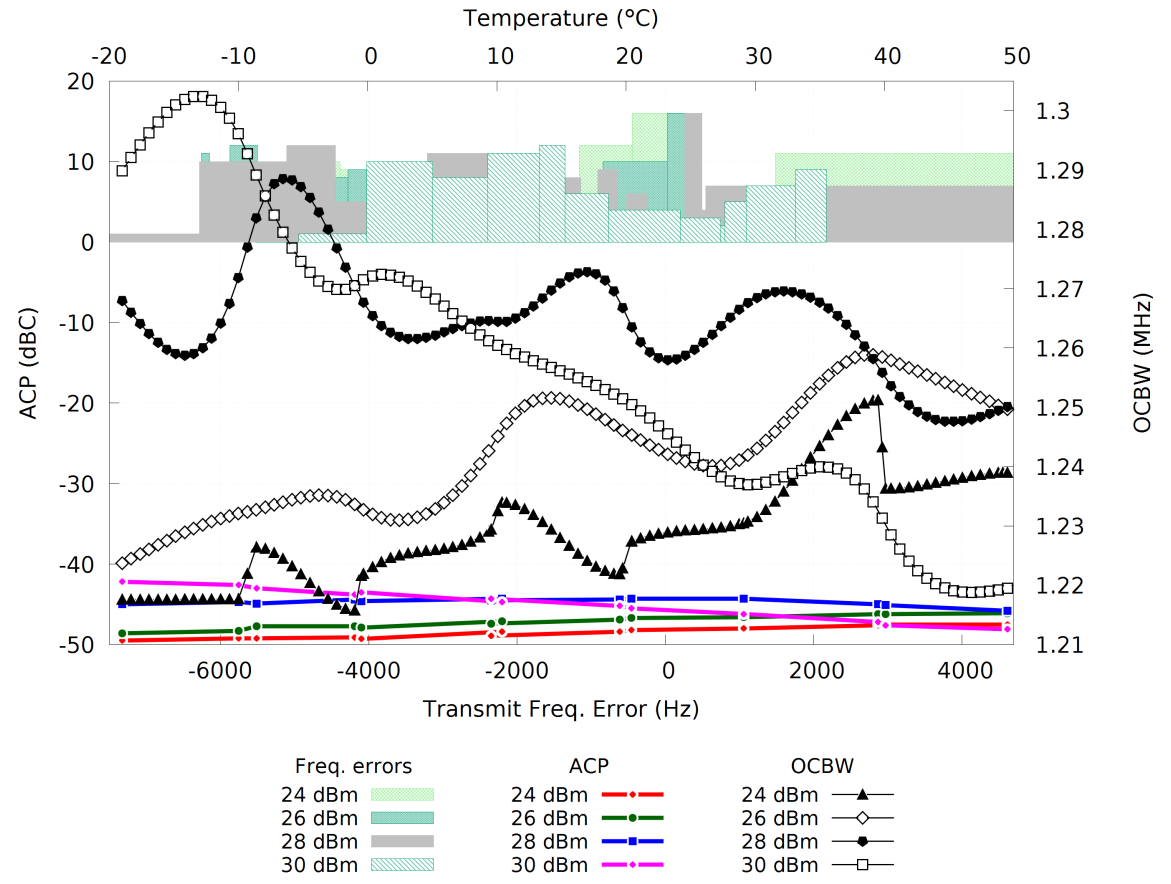

Figure 25: Relating jitter in frequency to the adjacent channel power and occupied bandwidth at $24 \mathrm{dBm}, 26 \mathrm{dBm}, 28 \mathrm{dBm}$ and $30 \mathrm{dBm}$ outputs during thermal cycle. 


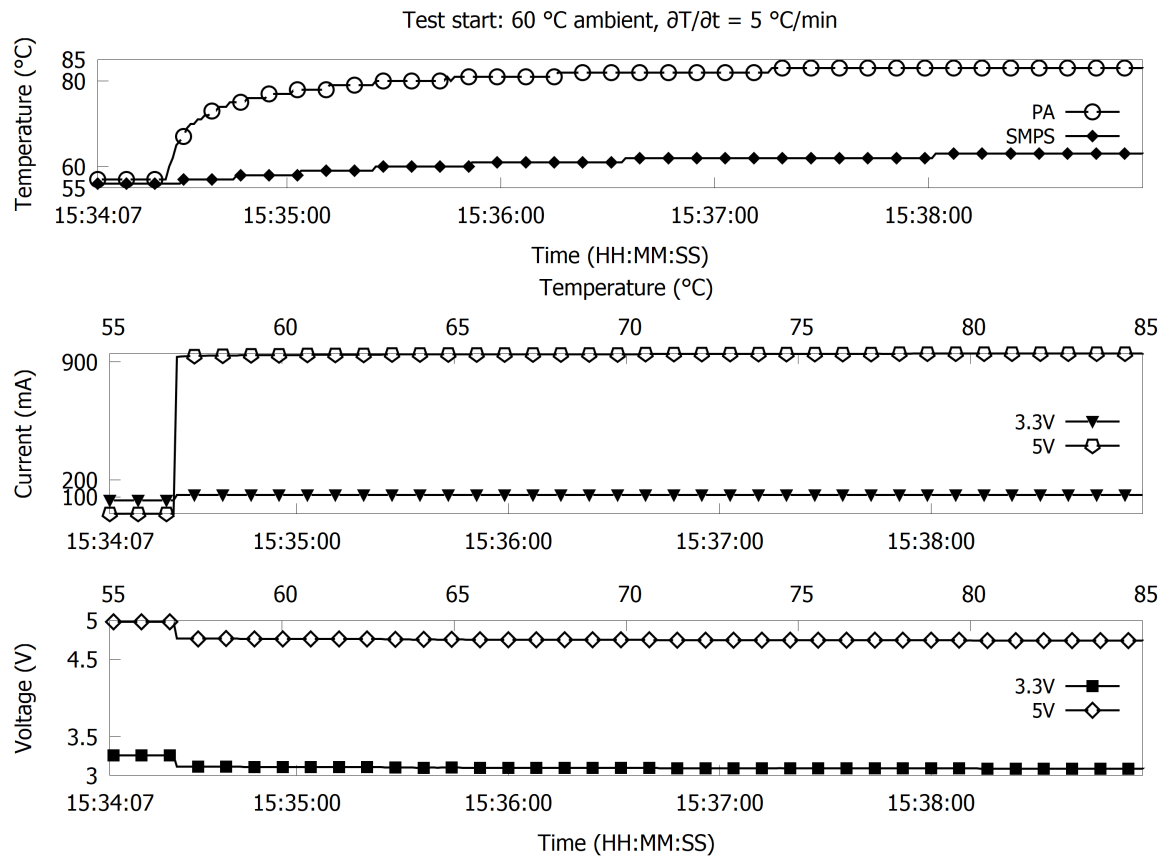

Figure 26: DC performance and supply stability in a 5 min thermal stress test.

\section{Accelerated Thermal Testing}

In addition to all the foregoing, a worse case accelerated thermal stress test is performed which shows robust stability in current, voltage and device temperatures. The steady-state plots of Fig. 26 is the accelerated test. The constancy of supply voltage over temperature guarantees that the active bias of the amplifier will not shift but only in response to the variable $\mathrm{RF}$ envelope voltage. The test begins at $60{ }^{\circ} \mathrm{C}$ and the temperature is rapidly increased to $84^{\circ} \mathrm{C}$ within $300 \mathrm{sec}$. The test gives confidence in the uncertainty margin of Fig. 2.

\section{Outlook}

Further work is anticipated toward qualification level testing. This is likely to include thermal vacuum bake out, possibly thermal vacuum cycling, vibration testing (sinusoid and random loading tests for the strongest possible acceleration profile of the various launch vehicles) and the radiation tests. 


\section{Implications and Conclusion}

If STX/C is used as a transmitter for the EO data, corruption in image download may require re-transmission which will happen in the next pass of the satellite that could be several hours to several days away depending on the orbit. In a survey of the first 100 CubeSats launched, $45 \%$ failed due to no contact with the satellite and, of the ones contacted, $17 \%$ failed later due to some fault in the communications systems (Swartwout, 2013). Therefore, the integrity of transmission is a vital criteria for the mission success.

Current estimates of the launch costs run around $50 \mathrm{US} \$ / \mathrm{g}-60 \mathrm{US} \$ / \mathrm{g}$ in $3 \mathrm{U}$ to $6 \mathrm{U}$ configurations (Launch, 2017). The vehicle and consequently the orbit selection could come after the negotiations and the baseline orbit/mission for which the hardware was designed may need tweaking late in the project. Furthermore, in a typical piggyback launch, the launch vehicle may drop off the satellite several tens of $\mathrm{km}$ away from the originally conceived orbit. Hence, the orbit propagation environment can be strikingly different than the environment in the concept mission definition. In the wake of such late decisions, the STX may be optimally reconfigured for the expected environment in power levels and in the RF front end. At the baseband level, even post-launch settings of the radio are possible after acquiring the orbit and averaging the thermal telemetry data of several orbits.

A rigorous thermo-functional treatment of $\mathrm{RF}$ transmission performance has been presented in a test-assisted approach. Two aspects of transmission are emphasized, viz., the modulation impairment and power amplification. Both concerns have been thermally validated for low noise and linear performance. The experimental data shows strong resilience to the temperatures, particularly, in low EVM, IQ gain imbalance, phase, frequency and magnitude errors as well as high $\rho$ and SNR. Measurements are related to the theory and implications of measurements on the nanosatellite mission are regularly touched. The performance of PA is stable and linear on the grounds of the compactness of channel bandwidth and the little splatter of energy. As an additional safety measure, a $15 \mathrm{~min}$ fail safe timeout disables the PA if it is accidentally left on by an uploaded telecommand, thereby safeguarding the amplifier from over-driving and de-rating for longer mission life. The timeout may be inhibited for longer transmissions or passes. Close scrutiny of the measurements reveal that in all errors the transmitter is more sensitive to $-10^{\circ} \mathrm{C}$ to $-20{ }^{\circ} \mathrm{C}$ range. The optimization of the STX/C RF circuits is focused at $28 \mathrm{dBm}$ at which the performance stands out from other levels 
over the full thermal cycle.

The transmission performance at the temperature boundaries outlined in the paper may assist in pre/post-launch calibration, calculating a better link budget, system level thermal testing on the full spacecraft, in protecting the on-board high-speed electronics and the magnetic devices for susceptibility, as well as for compliance of the frequency and power requirements, for transmission in the licensed bandwidth, levied by the spectrum allocation authority.

\section{Acknowledgment}

The authors would like to thank Axel Wottawa and Patrick Udhardt for test automation and Etnard Louw for thermal validation. We acknowledge ETSE Electronics for their role. Appreciation also goes to the anonymous reviewers for their insightful suggestions.

\section{References}

Angrisani, L., D’Apuzzo, M., \& Vadursi, M. 2006, Power measurement in digital wireless communication systems through parametric spectral estimation, IEEE Transactions on Instrumentation and Measurement, 55, 4, 1051-1058.

Aparin, V. 2001, Analysis of CDMA signal spectral regrowth and waveform quality, IEEE Transactions on Microwave Theory and Techniques, 49, 12, 2306-2314.

Bahl, I.J., Fundamentals of RF and microwave transistor amplifiers, John Wiley \& Sons (Hoboken, New Jersey, 2009).

Bouwmeester, J., \& Guo, J. 2010, Survey of worldwide pico- and nanosatellite missions, distributions and subsystem technology, Acta Astronautica, 67, 854-862.

Cho, M., Masui, H., Hatamura, T., Date, K., Horii, S., \& Obata, S. 2012, Overview of nano-satellite environmental tests standardization project: test campaign and standard draft, in Proceedings of 26th Annual AIAA/US Conference on Small Satellites, 1-11. 
Georgiadis, A. 2004, Gain, phase imbalance, and phase noise effects on error vector magnitude, IEEE Transactions on Vehicular Technology, 53, 2, 443449.

Gharaibeh, K.M., Gard, K.G., \& Steer, M.B. 2004, Accurate estimation of digital communication system metrics - SNR, EVM and $\rho$ in a nonlinear amplifier environment, in Proceedings of Microwave Measurements Conference, 64th ARFTG, 41-44.

Gilmore, D.G., Spacecraft thermal control handbook, vol. I, The Aerospace Press (El Segundo, California, 2002).

Grosch, T., Small signal microwave amplifier design, Noble Publishing Corporation (Atlanta, Georgia, 1999).

Josephson, G.C. 1970, On the definition and measurement of occupied bandwidth, IEEE Transactions on Electromagnetic Compatibility, EMC-12, 2, 33-37.

Launch Cost [Online], http://www.spaceflight.com/schedule-pricing

Mahmoud, H.A., \& Arslan, H. 2009, Error vector magnitude to SNR conversion for nondata-aided receivers, IEEE Transactions on Wireless Communications, 8, 5, 2694-2704.

McKinley, M.D., Remley, K.A., Myslinski, M., Kenney, J.S., Schreurs, D., \& Nauwelaers B. 2004, EVM calculation for broadband modulated signals, in Proceedings of Microwave Measurements Conference, 45-52.

Moer, W.V., Rolain, Y., \& Schoukens, J. 2002, An automatic harmonic selection scheme for measurements and calibration with the nonlinear vectorial network analyzer, IEEE Transactions on Instrumentation and Measurement, 51, 2, 337-341.

Nascetti, A., Pittella, E., Teofilatto, P., \& Pisa, S. 2015, High-gain S-band patch antenna system for earth-observation cubesat satellites, IEEE Antennas and Wireless Propagation Letters, 14, 434-437.

Noël, L., \& Brousse, P. 2011, Low-cost EVM test methodology for wireless transmitters applied to W-CDMA, IEEE Transactions on Instrumentation and Measurement, 60, 1, 170-175. 
Roblin, P., Nonlinear RF circuits and nonlinear vector network analyzers, Cambridge University Press (Cambridge, UK, 2011).

Rogers, J., \& Plett, C., Radio frequency integrated circuit design, Artech House (Norwood, Massachusetts, 2003).

Saleh, A.A.M., \& Cox, D.C. 1983, Improving the power-added efficiency of FET amplifiers operating with varying envelope signals, IEEE Transactions on Microwave Theory and Techniques, 31, 1, 51-56.

Sayre, C.W., Complete wireless design, 2nd edition, The McGraw-Hill Companies (New York, New York, 2008).

Scott, A.W. \& Frobenius, R., RF measurements for cellular phones and wireless data systems, John Wiley \& Sons (Hoboken, New Jersey, 2008).

Selva, D., \& Krejci, D. 2012, A survey and assessment of the capabilities of cubesats for earth observation, Acta Astronautica, 74, 50-68.

Steer, M., Microwave and RF design: a systems approach, SciTech Publishing (Raleigh, North Carolina, 2010).

Swartwout, M. 2013, The first one hundred cubesats: A statistical look, Journal of Small Satellites, 2, 2, 213-233.

Zhou, G.T., \& Raich, R. 2004, Spectral analysis of polynomial nonlinearity with applications to RF power amplifiers, EURASIP Journal on Applied Signal Processing, 12, 1831-1840. 